\title{
Light emission by point dipole located inside spherical (semiconductor) particle in the vicinity of a spherical metal particle
}

\author{
Yu.V. Kryuchenko and D.V. Korbutyak \\ V. Lashkaryov Institute of Semiconductor Physics, NAS of Ukraine \\ 41, prospect Nauky, 03028 Kyiv, Ukraine \\ Corresponding authore-mail:kdv45@isp.kiev.ua
}

\begin{abstract}
Proposed in this work is a theoretical model that enables to correctly calculate light emission characteristics of a hybrid nanosystem formed by a spherical semiconductor quantum dot (QD) and spherical metal nanoparticle (NP) when QD is excited by light. The QD that emits mainly dipole-type electromagnetic (EM) field can not be considered as a point dipole at small separation distances between QD and NP (of the same order as the QD size). However, only the "point dipole" concept of QD is used in all currently known theoretical models of hybrid nanosystems. Correspondingly, unlike a simulated "point dipole + spherical metal NP", the real hybrid nanosystem is nonspherical as a whole. In this work: i) relations have been obtained between the coefficients of EM field multipole expansions in two spherical coordinate systems with their origins in semiconductor QD and metal NP. As a result, spherical symmetry of semiconductor QD and metal NP can be used separately to solve the equations related to boundary conditions at their surfaces; ii) it has been shown that EM field emitted by QD can be represented as a sum of contributions of all crystal unit cells forming QD and that each unit cell can be considered as an emitting point dipole. The contribution of a particular unit cell to the total EM field is determined by the exciton wave function; iii) to be closer to the real situation, the contribution $\varepsilon_{i b}(\omega)$ of interband transitions to the NP permittivity has been taken into account in the visible spectral range.
\end{abstract}

Keywords: quantum dot, nanoparticle, hybrid nanostructure, point dipole, light emission.

Manuscript received 03.06.13; revised version received 05.08.13; accepted for publication 19.09.13; published online 30.09.13.

\section{Introduction}

In recent years, the number of theoretical and experimental studies of hybrid metal-semiconductor nanostructures has grown exponentially due to progress in fabrication techniques and wide prospects for efficient practical application of these nanostructures. The interest to hybrid nanostructures is caused by the fact that the excitonic, plasmonic and magnetic properties of their constituent parts can be combined in a single structure to give a synergistic effect. As a result, these nanostructures can possess a variety of new unique properties (for the latest review see e.g. [1]).

Under external laser excitation of a hybrid "semiconductor QD + metal NP" system, two possibilities of light absorption exist. At resonance or near-resonance excitation, light can be absorbed by both QD and NP (resonance means that the energies of laser photons are close to the energies of the localized surface plasmons in metal NP and the energies of size-quantized exciton states in QD), while at non-resonance excitation light is mainly absorbed in QD. In the first case, direct excitation of local surface plasmon modes in metal NP 
creates strong local fields in the NP vicinity enhancing optical energy absorption by QD. The QD emission of the absorbed energy in its turn is enhanced due to resonance between exciton states in QD and local radiating plasmon modes in metal NP, see e.g. [2]. This situation corresponds to the so-called "weak-coupling regime". In the "strong-coupling regime", resonant exciton-plasmon interactions modify exciton wave functions and local plasmon modes, thus leading to the changes in exciton and local plasmon resonance energies, see e.g. [3-6]. The case when quantum emitter (QD) is initially excited by external laser field also has been studied theoretically in a number of works, see e.g. [7]. However, in all currently known studies of the QD+NP nanosystems, QD as a whole is considered as a (point) dipole. Point dipole approximation is completely justified in the case of an isolated atom or luminophore molecule, or when the QD size is small enough as compared to the interparticle separation distance and NP size. However, it seems that at a comparable QD size and interparticle separation distance, light emission by QD should be considered more accurately. The goal of this work is to develop a model allowing to correctly calculate semiconductor QD emission in the vicinity of metal NP. The key point of the proposed theory is the statement that despite semiconductor QD as a whole cannot be considered as a point dipole at comparable sizes and distances in a QD+NP nanosystem, its constituent parts, i.e. crystal unit cells forming QD can be. Therefore, to calculate the emission characteristics of semiconductor QD in the vicinity of metal NP, it is necessary to consider previously the contribution of an arbitrary unit cell of QD, and then integrate the contributions of all the unit cells with corresponding weight factors determined by the exciton wave function. In this work, we consider the first part of this problem that is reduced to the calculation of an EM field of a point dipole radiating inside semiconductor QD in the case when QD is located in the immediate vicinity of metal NP.

\section{Model validation}

In this section, we schematically present grounds for considering QD as an ensemble of oscillating point dipoles inside QD. Consider the multi-electron system of semiconductor QD when one of the electrons is excited from the state associated with the valence band of bulk material that forms QD into the state associated with the conduction band. In the linear approximation, the solution of the time-dependent Schrödinger equation can be written in this case as follows:

$$
\Psi(t)=\Psi_{0} \exp \left(-i \omega_{0} t\right)+\Psi_{1} \exp \left(-i\left(\omega_{0}+\omega\right) t\right),
$$

where $\Psi_{0}$ is the wave-function of the ground state of the multi-electron system with Hartree-Fock energy $\hbar \omega_{0}$, $\Psi_{1}$ - correction to the wave function $\Psi_{0}$ caused by specified perturbation, $\hbar \omega$ - energy of perturbation. The wave function $\Psi_{0}$ can be written as antisymmetrized product of one-electron orthonormal Wannier lattice-site functions of the valence band [8]:

$$
\begin{aligned}
& \Psi_{0}\left(\widetilde{\xi}_{1}, \ldots \widetilde{\xi}_{N K_{\alpha \sigma}}\right)=1 / \sqrt{\left(N K_{\alpha \sigma}\right) !} \sum_{p}(-1)^{A_{p}} \times \\
& \times \hat{p} \prod_{\widetilde{\mathbf{l}}, \alpha, \sigma} b_{\widetilde{\mathbf{l}}, \alpha, \sigma}^{V}(. .)\left\{\tilde{\xi}_{1}, \ldots, \widetilde{\xi}_{N K_{\alpha \sigma}}\right\},
\end{aligned}
$$

where $\hat{p}$ is the operator of a particular permutation of the total $\left(N K_{\alpha \sigma}\right)$ ! permutations, at which $N K_{\alpha \sigma}$ spacespin electron variables $\left\{\widetilde{\xi}_{1}, \widetilde{\xi}_{2}, \ldots, \widetilde{\xi}_{N K_{\alpha \sigma}}\right\} \quad$ are distributed over $N K_{\alpha \sigma}$-fold product of the valence band lattice-site Wannier functions $b_{\widetilde{\mathbf{n}}, \alpha, \sigma}^{V}(\widetilde{\xi})=b_{\widetilde{\mathbf{n}}, \alpha}^{V}(\widetilde{\mathbf{r}}) \chi_{\sigma}(s)$ as their arguments ( $\widetilde{\mathbf{r}}$ is the spatial and $s$ is the spin variables), $A_{p}$ is multiplicity of the permutation $p, N-$ total number of lattice sites (unit cells) in QD, $\tilde{\mathbf{n}}-$ lattice site coordinate, $\widetilde{\mathbf{l}}$ in (2) runs over all $N$ lattice sites of QD, $\sigma$ is a symbol characterizing electron spin state, $\alpha$ - symbol characterizing crystallographic directions (in crystals with cubic lattice structure, valence band Wannier states are degenerated over all three crystallographic directions), $K_{\alpha, \sigma}$ is the multiplicity of degeneracy over the electron spin and crystallographic directions. Here and below, the tilde over the variables means that they are written in the coordinate system centered in semiconductor QD.

The wave function $\Psi_{1}$ can be written as a superposition of antisymmetrized $\chi_{\widetilde{\mathbf{s}} \widetilde{\mathbf{t}}}^{\sigma \sigma^{\prime} \alpha}$-states in which one of the electrons of the multi-electron system is transferred from the localized in the site $\widetilde{\mathbf{s}} \alpha \sigma$-Wannier state of the valence band $b_{\widetilde{\mathbf{s}}, \alpha, \sigma}^{V}$ into the localized in the site $\tilde{\mathbf{t}} \quad \sigma^{\prime}$-Wannier state of the conduction band $b_{\widetilde{\mathbf{t}}, \sigma^{\prime}}^{C}$ : $\Psi_{1}^{\sigma \sigma^{\prime}}\left(\widetilde{\xi}_{1}, \ldots, \widetilde{\xi}_{N K_{\alpha \sigma}}\right)=\sum_{\widetilde{\mathbf{s}}, \widetilde{\mathbf{t}}, \alpha} C_{\widetilde{\mathbf{s}} \widetilde{\mathbf{t}}}^{\sigma \sigma^{\prime} \alpha} \chi_{\widetilde{\mathbf{s}} \tilde{\mathbf{t}}}^{\sigma \sigma^{\prime} \alpha}\left(\widetilde{\xi}_{1}, \ldots, \widetilde{\xi}_{N K_{\alpha \sigma}}\right) .(3)$

The coefficients $C_{\widetilde{\mathbf{s}} \widetilde{\mathbf{t}}}^{\sigma \sigma^{\prime} \alpha}$ are the amplitudes of these $\chi_{\widetilde{\mathbf{s}} \widetilde{\mathbf{t}}}^{\sigma \sigma^{\prime} \alpha}$-states,

$$
\begin{aligned}
& \chi_{\widetilde{\mathbf{s}} \widetilde{\mathbf{t}}}^{\sigma \sigma^{\prime} \alpha}=1 / \sqrt{\left(N K_{\alpha \sigma}\right) !} \sum_{p}(-1)^{A_{p}} \hat{p} b_{\widetilde{\mathbf{t}}, \sigma^{\prime}}^{C}(. .) \times \\
& \times \prod_{\widetilde{\mathbf{1}}, \gamma, \eta \neq \widetilde{\mathbf{s}}, \alpha, \sigma} b_{\widetilde{\mathbf{l}}, \gamma, \eta}^{V}(. .)\left\{\tilde{\xi}_{1}, \ldots, \widetilde{\xi}_{N K_{\alpha \sigma}}\right\} .
\end{aligned}
$$

Similarly to $[9,10]$, introduce the mean dipole moment $\mathbf{P}(\widetilde{\mathbf{L}}, t)$ of the lattice site $\tilde{\mathbf{L}}$ generated due to the above specified perturbation of the QD multielectron system,

$$
\mathbf{P}(\tilde{\mathbf{L}}, t)=\int \Psi^{*}(t) \hat{p}_{0}(\tilde{\mathbf{L}}) \Psi(t) d \widetilde{\Omega}_{e},
$$

where integration is taken over all $N K_{\alpha \sigma}$ variables of 
the multi-electron system, $\hat{p}_{0}(\tilde{\mathbf{L}})$ is the dipole moment operator in the site $\widetilde{\mathbf{L}}$,

$$
\hat{p}_{0}(\tilde{\mathbf{L}})=e \sum_{i}\left(\widetilde{\mathbf{r}}_{i}-\tilde{\mathbf{L}}\right) T\left(\widetilde{\mathbf{r}}_{i}-\tilde{\mathbf{L}}\right) .
$$

In this formula, summation is taken over all $N$ spatial variables of the multi-electron system, $T\left(\widetilde{\mathbf{r}}_{i}-\widetilde{\mathbf{L}}\right)=0$, if spatial variable $\widetilde{\mathbf{r}}_{i}$ turns out to be outside the unit cell at the site $\widetilde{\mathbf{L}}$, and $T\left(\widetilde{\mathbf{r}}_{i}-\widetilde{\mathbf{L}}\right)=1$, if $\widetilde{\mathbf{r}}_{i}$ is within the unit cell.

By carrying out integration in (5), the following expression can be obtained:

$$
\mathbf{P}(\widetilde{\mathbf{L}}, t)=e \sum_{\widetilde{\mathbf{s}}, \widetilde{\mathbf{t}}, \alpha} C_{\widetilde{\mathbf{s}} \widetilde{\mathbf{t}}}^{\alpha} \int_{V_{0}(\widetilde{\mathbf{L}})} b_{\widetilde{\mathbf{t}}}^{C^{*}}(\widetilde{\mathbf{r}})(\widetilde{\mathbf{r}}-\widetilde{\mathbf{L}}) b_{\widetilde{\mathbf{s}}, \alpha}^{V}(\widetilde{\mathbf{r}}) d^{3} \widetilde{r} \exp (-i \omega t) .(7)
$$

The integral in (7) is taken over the volume $V_{0}$ of the unit cell at the site $\widetilde{\mathbf{L}}$. Since Wannier functions are strongly localized within the respective unit cells at the sites $\widetilde{\mathbf{s}}$ and $\widetilde{\mathbf{t}}$, the only option when integral in (7) is not zero corresponds to the case $\widetilde{\mathbf{s}}=\widetilde{\mathbf{t}}=\widetilde{\mathbf{L}}$. By introducing the notation $\mathbf{p}_{\alpha}$ for the independent of a particular site $\widetilde{\mathbf{s}}$ unit cell dipole moment,

$$
\mathbf{p}_{\alpha}=e \int_{V_{0}(\widetilde{\mathbf{s}})} b_{\widetilde{C^{*}}}^{C^{*}}(\widetilde{\mathbf{r}}) \widetilde{\mathbf{r}} b_{\widetilde{\mathbf{s}}, \alpha}^{V}(\widetilde{\mathbf{r}}) d^{3} \widetilde{r},
$$

the following expression for the mean dipole moment $\mathbf{P}(\widetilde{\mathbf{L}}, t)$ of the lattice site $\widetilde{\mathbf{L}}$ can be obtained:

$\mathbf{P}(\widetilde{\mathbf{L}}, t)=\sum_{\alpha} C_{\widetilde{\mathbf{L}} \widetilde{\mathbf{L}}}^{\alpha} \mathbf{p}_{\alpha} \exp (-i \omega t)$,

i.e. $\mathbf{P}(\tilde{\mathbf{L}}, t)$ is formed by the unit cell dipole moments $\mathbf{p}_{\alpha}$ with weight coefficients $C_{\tilde{\mathbf{L}} \widetilde{\mathbf{L}}}^{\alpha}$. The polarization density due to the generated dipole moment $\mathbf{p}_{\alpha}$ in the unit cell $\tilde{\mathbf{L}}$ is $\mathbf{P}_{\alpha}(\tilde{\mathbf{L}})=\mathbf{p}_{\alpha} / V_{0}$. By passing from the discrete variable $\widetilde{\mathbf{L}}$ to continuous $\widetilde{\mathbf{r}}_{d}$, the discrete amplitude $C_{\widetilde{\mathbf{L}} \widetilde{\mathbf{L}}}^{\alpha}$ transforms into the exciton envelope wave function $\Psi_{e x}^{\alpha}\left(\widetilde{\mathbf{r}}_{d}, \widetilde{\mathbf{r}}_{d}\right)$ in electron-electron representation. In the first approximation, this function takes a form of the product of electron and hole wave functions of size-quantized states in QD, $\Psi_{e x}^{\alpha}\left(\widetilde{\mathbf{r}}_{d}, \widetilde{\mathbf{r}}_{d}\right)=\varphi_{e}\left(\widetilde{\mathbf{r}}_{d}\right) \varphi_{h}^{\alpha *}\left(\widetilde{\mathbf{r}}_{d}\right)$. Accordingly, the located in the site $\widetilde{\mathbf{L}}$ unit cell dipole with the dipole moment $\mathbf{p}_{\alpha}$ (8) transforms into the located in the point $\widetilde{\mathbf{r}}_{d}$ point dipole with the same dipole moment $\mathbf{p}_{\alpha}$, the polarization density $\mathbf{P}_{\alpha}(\tilde{\mathbf{L}})$ transforms into $\mathbf{P}_{\alpha}(\widetilde{\mathbf{r}})=\mathbf{p}_{\alpha} \delta\left(\widetilde{\mathbf{r}}-\widetilde{\mathbf{r}}_{d}\right)$. By summing up the contributions of all the unit cells of QD to the total EM field, the discrete sums of the type $\sum_{\widetilde{\mathbf{L}}} C_{\widetilde{\mathbf{L}} \widetilde{\mathbf{L}}}^{\alpha} f(\widetilde{\mathbf{L}})$ should be replaced by the integrals $\int \Psi_{e x}^{\alpha}\left(\widetilde{\mathbf{r}}_{d}, \widetilde{\mathbf{r}}_{d}\right) f\left(\widetilde{\mathbf{r}}_{d}\right) d^{3} \widetilde{r}_{d}$.

\section{System under consideration}

Consider the system formed by the following three constituents (see Fig. 1):

1) Spherical metal NP of the radius $R_{1}$ which is characterized by a non-local permittivity $\varepsilon_{1}(\omega, k)$. With account of spatial dispersion as well as additional electron scattering at the NP walls, one can consider NP of extremely small sizes (less than $10 \mathrm{~nm}$ in diameter) by using the methods of classical macroscopic electrodynamics [11]. In the optical range of frequencies, $\varepsilon_{1}(\omega, k)$ can be expressed as

$\varepsilon_{1}^{T(L)}(\omega, k)=1+\varepsilon_{i b}(\omega)+\varepsilon_{p l}^{T(L)}(\omega, k)$,

where the second term in the right-hand side is the contribution of bound electrons participating in interband electronic transitions, and the third term

$\varepsilon_{p l}^{T(L)}(\omega, k)=-\frac{\omega_{p}^{2}}{\omega\left[\omega+i\left(\Gamma+A v_{\mathrm{F}} / R_{1}\right)\right]-\beta_{T(L)}^{2} k^{2}}$

is the contribution of free electrons. Indices $T$ and $L$ are used to denote transverse $(T)$ and longitudinal $(L)$ components of the permittivity. Within the hydrodynamic model $\beta_{L}=\sqrt{3 / 5} v_{\mathrm{F}}$, where $v_{\mathrm{F}}$ is the Fermi velocity $\left(v_{\mathrm{F}} \approx 1.4 \cdot 10^{8} \mathrm{~cm} / \mathrm{s}\right.$ in the case of gold or silver), while $\beta_{T}=0$ and therefore $\varepsilon_{1}^{T}(\omega, k) \equiv \varepsilon_{1}^{T}(\omega, 0)$. The decay constant $\Gamma \approx v_{\mathrm{F}} / l_{f}$, where $l_{f}$ is the mean free path of electrons, the constant $A$ takes values within the range from 0.1 to 0.7 [11], depending on the mechanism of electron scattering on the NP walls and other factors, $\omega_{p}^{2}=4 \pi n e^{2} / m^{*}$ is the plasma frequency

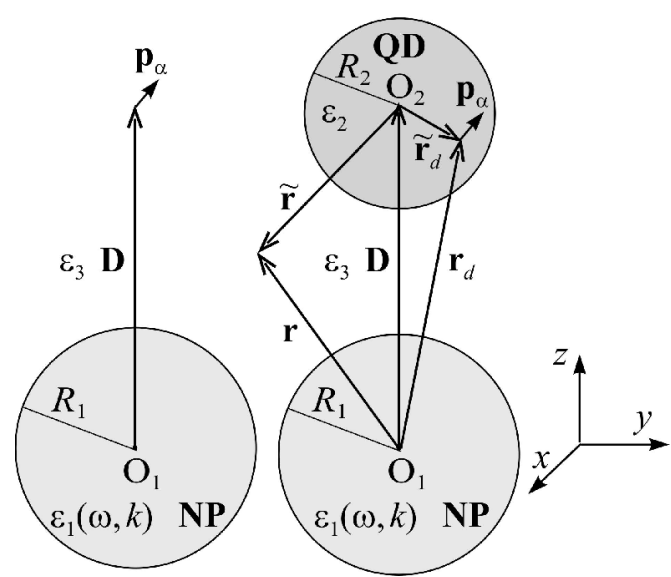

Fig. 1. Point dipole $\mathbf{p}_{\alpha}$ in the vicinity of metal NP; the system is spherically symmetric (a). Semiconductor QD with point dipole $\mathbf{p}_{\alpha}$ in the vicinity of metal NP; the system isn't spherically symmetric (b). 
of the NP material. At a given frequency $\omega$, the wave number $k_{T}$ of transverse electromagnetic excitations in $\mathrm{NP}$ is determined by the dispersion law $k_{T}^{2}=\varepsilon_{1}^{T}(\omega, 0) \omega^{2} / c^{2}$, while the wave number $k_{L}$ of the longitudinal electric excitations can be found from the condition $\varepsilon_{1}^{L}\left(\omega, k_{L}\right)=0$.

2) Spherical semiconductor $\boldsymbol{Q D}$ of the radius $R_{2}$ with a background permittivity $\varepsilon_{2}$. The distance between QD and NP centers is $D>R_{1}+R_{2}$.

3) Barrier matrix with the permittivity $\varepsilon_{3}$ in which QD and NP are embedded.

The formulated electrodynamic problem can be considered using multipole expansions of EM field. Two the most known forms of the multipole expansions are those presented in [12] and [13]. In the Jackson textbook [12], vector spherical harmonics $\mathbf{X}_{l m}(\Omega)$ are used for this purpose. The expansions on the base of spherical vector wave functions $\mathbf{M}_{l m}^{1(3)}(r, \Omega)$ and $\mathbf{N}_{l m}^{1(3)}(r, \Omega)$ are presented in the Stratton textbook [13]. In fact, these two forms of expansions are completely equivalent. However, to make intermediate calculations less cumbersome, the Jackson formalism is used below. Correspondingly, all further formulas are written in the Gaussian unit system in compliance with [12].

When calculating the EM field in the QD+NP system, the boundary conditions on both QD and NP spherical surfaces should be satisfied. To ensure this, it is necessary to establish a relation between multipole components of the field in two different spherical coordinate systems with their origins $\mathrm{O}_{1}$ in metal NP and $\mathrm{O}_{2}$ in semiconductor QD.

\section{Relationships between multipole expansions of electromagnetic field in two coordinate systems shifted to each other}

When calculating the EM field in a nonspherical QD+NP system, it's desirable to use spherical symmetry of QD and NP separately. In accordance with [12], in the $n$-th material with the permittivity $\varepsilon_{n}$, the transversal electric and magnetic fields can be expressed as follows:

$\mathbf{E}_{n}(\mathbf{r})=\sum_{l=1}^{\infty} \sum_{m=-1}^{l}\left\{\frac{i}{k_{0} \varepsilon_{n}} a_{n, E}(l, m) \nabla \times\left[f_{l}\left(k_{n} r\right) \mathbf{X}_{l m}(\Omega)\right]+\right.$

$\left.+a_{n, M}(l, m) f_{l}\left(k_{n} r\right) \mathbf{X}_{l m}(\Omega)\right\}$,

$\mathbf{B}_{n}(\mathbf{r})=\sum_{l=1}^{\infty} \sum_{m=-1}^{l}\left\{a_{n, E}(l, m) f_{l}\left(k_{n} r\right) \mathbf{X}_{l m}(\Omega)-\right.$

$\left.-\frac{i}{k_{0}} a_{n, M}(l, m) \nabla \times\left[f_{l}\left(k_{n} r\right) \mathbf{X}_{l m}(\Omega)\right]\right\}$,

where $\quad k_{0}=\omega / c, \quad k_{n}=k_{0} \sqrt{\varepsilon_{n}}, \quad a_{n, E}(l, m) \quad$ and $a_{n, M}(l, m)$ are the multipole amplitudes (coefficients) of the electric and magnetic types, respectively, $f_{l}(x)$ is the first kind spherical Hankel function $h_{l}^{(1)}(x)$ denoted hereafter as $h_{l}(x)$, or the spherical Bessel function $j_{l}(x)$, or their combination, depending on the region of space and asymptotic or boundary conditions that should be satisfied, symbol $\Omega$ denotes angular variables $\{\theta, \varphi\}$. Normalized vector spherical harmonics $\mathbf{X}_{l m}(\Omega)$ have the following explicit form:

$$
\begin{aligned}
& \mathbf{X}_{l m}(\Omega)=\frac{r}{i \sqrt{l(l+1)}}\left[\mathbf{e}_{r} \times \nabla Y_{l m}(\Omega)\right]= \\
& =\frac{1}{i \sqrt{l(l+1)}}\left[-\mathbf{e}_{\theta} \frac{1}{\sin \theta} \frac{\partial}{\partial \varphi}+\mathbf{e}_{\varphi} \frac{\partial}{\partial \theta}\right] Y_{l m}(\Omega),
\end{aligned}
$$

where

$Y_{l m}(\Omega)=[(2 l+1)(l-m) ! /(4 \pi(l+m) !)]^{1 / 2} P_{l}^{m}(\cos \theta) \exp (\operatorname{im} \varphi)$ are the normalized scalar spherical harmonics, $P_{l}^{m}(x)$ are the associated Legendre functions. Accordingly,

$$
\begin{aligned}
& \nabla \times\left[f_{l}\left(k_{n} r\right) \mathbf{X}_{l m}(\Omega)\right]=\frac{i}{\sqrt{l(l+1)}} \times \\
& \times\left\{l(l+1) \frac{f_{l}\left(k_{n} r\right)}{r} Y_{l m}(\Omega) \mathbf{e}_{r}+\frac{d\left(r f_{l}\left(k_{n} r\right)\right)}{d r} \nabla Y_{l m}(\Omega)\right\}= \\
& =\frac{i}{r \sqrt{l(l+1)}}\left\{l(l+1) f_{l}\left(k_{n} r\right) Y_{l m}(\Omega) \mathbf{e}_{r}+\right. \\
& \left.+\frac{d\left(r f_{l}\left(k_{n} r\right)\right)}{d r}\left[\mathbf{e}_{\theta} \frac{\partial}{\partial \theta}+\mathbf{e}_{\varphi} \frac{1}{\sin \theta} \frac{\partial}{\partial \theta}\right] Y_{l m}(\Omega)\right\} .
\end{aligned}
$$

In (14) and (15), $\mathbf{e}_{r}, \mathbf{e}_{\theta}$ and $\mathbf{e}_{\varphi}$ are the orts of the spherical coordinate system. By further consideration, the following orthogonality and normalization conditions are exploited:

$$
\begin{aligned}
& \int Y_{l^{\prime} m^{\prime}}^{*}(\Omega) \cdot Y_{l m}(\Omega) d \Omega=\delta_{l l^{\prime}} \delta_{m m^{\prime}} \\
& \int \mathbf{X}_{l^{\prime} m^{\prime}}^{*}(\Omega) \cdot \mathbf{X}_{l m}(\Omega) d \Omega=\delta_{l l^{\prime}} \delta_{m m^{\prime}} \\
& \int\left[\nabla \times \mathbf{X}_{l^{\prime} m^{\prime}}^{*}(\Omega)\right] \cdot\left[\nabla \times \mathbf{X}_{l m}(\Omega)\right] d \Omega=\delta_{l l^{\prime}} \delta_{m m^{\prime}} \frac{l(l+1)+1}{r^{2}} \\
& \int \nabla Y_{l^{\prime} m^{\prime}}^{*}(\Omega) \cdot \nabla Y_{l m}(\Omega) d \Omega=\delta_{l l^{\prime}} \delta_{m m^{\prime}} \frac{l(l+1)}{r^{2}} \\
& \int \mathbf{X}_{l^{\prime} m^{\prime}}^{*}(\Omega) \cdot\left[\nabla \times \mathbf{X}_{l m}(\Omega)\right] d \Omega=0 \\
& \int \mathbf{X}_{l^{\prime} m^{\prime}}^{*}(\Omega) \cdot \nabla Y_{l m}(\Omega) d \Omega=0 \\
& \int_{-1}^{1} P_{l^{\prime}}^{m}(x) P_{l}^{m}(x) d x=\delta_{l l^{\prime}} \frac{2}{2 l+1} \frac{(l+m) !}{(l-m) !}
\end{aligned}
$$

Using the analytical approach outlined in Chapter 16 of the Jackson textbook [12], it can be found that for the oscillating point dipole $p_{\alpha} \mathbf{n}_{\alpha} \exp (-i \omega t)$ located at a 
point $\mathbf{r}_{d}$ ( $\mathbf{n}_{\alpha}$ is a unit vector along the dipole direction), the multipole coefficients $a_{n, E}(l, m)$ and $a_{n, M}(l, m)$ in the expansions (12) and (13) should take the following form:

$$
a_{n, E}^{d}(l, m)=4 \pi p_{\alpha} k_{n} k_{0} \mathbf{n}_{\alpha} \cdot\left[\nabla_{\mathbf{r}_{d}} \times g_{l}\left(k_{n} r_{d}\right) \mathbf{X}_{l m}^{*}\left(\Omega_{d}\right)\right],
$$

$a_{n, M}^{d}(l, m)=4 \pi i p_{\alpha} k_{n} k_{0}^{2} g_{l}\left(k_{n} r_{d}\right) \mathbf{n}_{\alpha} \cdot \mathbf{X}_{l m}^{*}\left(\Omega_{d}\right)$,

and in this case the following correlation between functions $f_{l}\left(k_{n} r\right)$ in (12) and (13) and $g_{l}\left(k_{n} r_{d}\right)$ in (23) and (24) should take place:

$$
\begin{aligned}
& f_{l}\left(k_{n} r\right)=j_{l}\left(k_{n} r\right), \\
& g_{l}\left(k_{n} r_{d}\right)=h_{l}\left(k_{n} r_{d}\right), \text { if } r<r_{d}, \\
& f_{l}\left(k_{n} r\right)=h_{l}\left(k_{n} r\right), \\
& g_{l}\left(k_{n} r_{d}\right)=j_{l}\left(k_{n} r_{d}\right), \text { if } r>r_{d} .
\end{aligned}
$$

In fact, these multipole expansions of the point dipole field coincide with those expressed in spherical vector wave functions $\mathbf{M}_{l m}^{1(3)}(r, \Omega)$ and $\mathbf{N}_{l m}^{1(3)}(r, \Omega)$ [14]. For convenience of further consideration, introduce the following notations:

$$
a_{n, E}^{d,>}(l, m)=4 \pi p_{\alpha} k_{n} k_{0} \mathbf{n}_{\alpha} \cdot\left[\nabla_{\mathbf{r}_{d}} \times j_{l}\left(k_{n} r_{d}\right) \mathbf{X}_{l m}^{*}\left(\Omega_{d}\right)\right],
$$

$a_{n, E}^{d,<}(l, m)=4 \pi p_{\alpha} k_{n} k_{0} \mathbf{n}_{\alpha} \cdot\left[\nabla_{\mathbf{r}_{d}} \times h_{l}\left(k_{n} r_{d}\right) \mathbf{X}_{l m}^{*}\left(\Omega_{d}\right)\right]$,

$a_{n, M}^{d,>}(l, m)=4 \pi i p_{\alpha} k_{n} k_{0}^{2} j_{l}\left(k_{n} r_{d}\right) \mathbf{n}_{\alpha} \cdot \mathbf{X}_{l m}^{*}\left(\Omega_{d}\right)$,

$$
a_{n, M}^{d,<}(l, m)=4 \pi i p_{\alpha} k_{n} k_{0}^{2} h_{l}\left(k_{n} r_{d}\right) \mathbf{n}_{\alpha} \cdot \mathbf{X}_{l m}^{*}\left(\Omega_{d}\right) .
$$

\section{A. Homogeneous medium}

First, consider a point dipole in a homogeneous system $\left(\varepsilon_{1}=\varepsilon_{2}=\varepsilon_{3}\right)$. According to (12), multipole expansions of the dipole electric field in two shifted to each other spherical coordinate systems with their origins at $\mathrm{O}_{1}$ and $\mathrm{O}_{2}$ points (see Fig. 1) should have the following form:

$\mathbf{E}_{3}(\mathbf{r})=\sum_{l, \mu}\left\{\frac{i}{k_{0} \varepsilon_{3}} a_{3, E}(l, \mu)\left[\nabla \times f_{l}\left(k_{3} r\right) \mathbf{X}_{l \mu}(\Omega)\right]+\right.$

$\left.+a_{3, M}(l, \mu) f_{l}\left(k_{3} r\right) \mathbf{x}_{l \mu}(\Omega)\right\}$,

$\mathbf{E}_{3}(\widetilde{\mathbf{r}})=\sum_{l, \mu}\left\{\frac{i}{k_{0} \varepsilon_{3}} \widetilde{a}_{3, E}^{d}(l, \mu)\left[\nabla_{\widetilde{\mathbf{r}}} \times f_{l}\left(k_{3} \widetilde{r}\right) \mathbf{X}_{l \mu}(\widetilde{\Omega})\right]_{+}\right.$

$\left.+\widetilde{a}_{3, M}^{d}(l, \mu) f_{l}\left(k_{3} \tilde{r}\right) \mathbf{X}_{l \mu}(\widetilde{\Omega})\right\}$.

Remember that variables and quantities with tilde are those related to spherical coordinate system with its origin at $\mathrm{O}_{2}$ point. Electric field is independent on a particular coordinate system used for its presentation. For this reason, the fields (31) and (32) are identically equal to each other in an arbitrary point of the space, $\mathbf{E}_{3}(\mathbf{r}) \equiv \mathbf{E}_{3}(\widetilde{\mathbf{r}})$. Being multiplied by $\mathbf{X}_{n m}^{*}(\Omega)$ and integrated over solid angle $\Omega$ with account of the explicit expressions (14) and (15) and normalization and orthogonality conditions (17) and (20), this identity transforms into the following relationship between the coefficient $a_{3, M}(n, m)$ on the one hand, and the coefficients $\widetilde{a}_{3, M}^{d}(l, m)$ and $\widetilde{a}_{3, E}^{d}(l, m)$ on the other hand:

$a_{3, M}(n, m)=\sum_{l=1}^{\infty}\left[F_{n l}^{m}(D) \widetilde{a}_{3, M}^{d}(l, m)+\right.$

$\left.+m\left(k_{3} D / \sqrt{\varepsilon_{3}}\right) G_{n l}^{m}(D) \widetilde{a}_{3, E}^{d}(l, m)\right]$.

Analogously, from the identity $\mathbf{B}_{3}(\mathbf{r}) \equiv \mathbf{B}_{3}(\widetilde{\mathbf{r}})$ of the magnetic fields (13) written in two shifted spherical coordinate systems, the following relationship between the coefficient $a_{3, E}(n, m)$ and coefficients $\widetilde{a}_{3, M}^{d}(l, m)$ and $\widetilde{a}_{3, E}^{d}(l, m)$ can be obtained:

$$
\begin{aligned}
& a_{3, E}(n, m)=\sum_{l=1}^{\infty}\left[F_{n l}^{m}(D) \widetilde{a}_{3, E}^{d}(l, m)-\right. \\
& \left.-m\left(k_{3} D\right) \sqrt{\varepsilon_{3}} G_{n l}^{m}(D) \widetilde{a}_{3, M}^{d}(l, m)\right] .
\end{aligned}
$$

In (33) and (34), $F_{n l}^{m}(D)$ and $G_{n l}^{m}(D)$ are the following linking coefficients:

$$
\begin{aligned}
& F_{n l}^{m}(D)=\frac{R_{n l}^{|m|}}{f_{n}\left(k_{3} r\right)} \int_{-1}^{1} P_{n}^{|m|}(x)\left[l(l+1) \frac{f_{l}\left(k_{3} \widetilde{r}\right)}{s^{2}}\left(1-x \frac{D}{r}\right) \times\right. \\
& \left.\times P_{l}^{|m|}(\widetilde{x})+\left(k_{3} D\right)\left(\frac{f_{l}\left(k_{3} \widetilde{r}\right)}{k_{3} \widetilde{r}}+f_{l}^{\prime}\left(k_{3} \widetilde{r}\right)\right)\left(1-\widetilde{x}^{2}\right) \frac{d P_{l}^{|m|}(\widetilde{x})}{d \widetilde{x}}\right] d x,
\end{aligned}
$$

$G_{n l}^{m}(D)=\frac{R_{n l}^{|m|}}{f_{n}\left(k_{3} r\right)} \int_{-1}^{1} f_{l}\left(k_{3} \widetilde{r}\right) P_{n}^{|m|}(x) P_{l}^{|m|}(\widetilde{x}) d x$,

where

$$
R_{n l}^{m}=\frac{1}{2} \sqrt{\frac{2 n+1}{n(n+1)} \frac{(n-m) !}{(n+m) !} \frac{2 l+1}{l(l+1)} \frac{(l-m) !}{(l+m) !}},
$$

$$
f^{\prime}(y) \text { is a derivative } d f(y) / d y \text {, }
$$
$s=\left[1+(D / r)^{2}-2 x D / r\right]^{1 / 2}, \quad \tilde{x}=(x-D / r) / s, \tilde{r}=r s$. To obtain expressions (35) and (36), the original integrals are integrated by parts with account of $f_{l}(z)$ and $P_{l}^{m}(x)$ properties. Besides, it is also taken into account that $\mathbf{e}_{\widetilde{\varphi}} \equiv \mathbf{e}_{\varphi}$,

$$
\begin{aligned}
& \mathbf{e}_{\widetilde{r}}=\left[(1-x D / r) \mathbf{e}_{r}+\sqrt{1-x^{2}}(D / r) \mathbf{e}_{\theta}\right] / s, \\
& \mathbf{e}_{\widetilde{\theta}}=\left[-\sqrt{1-x^{2}}(D / r) \mathbf{e}_{r}+(1-x D / r) \mathbf{e}_{\theta}\right] / s .
\end{aligned}
$$


The linking coefficients $F_{n l}^{m}$ and $G_{n l}^{m}$ look like coordinate-dependent ones. However, they don't really depend on the "linking coordinate" $r$. It can be easily checked by numerical integration. In the region $\tilde{r}>\widetilde{r}_{d}$ (see Fig. 1), the function $f_{l}\left(k_{3} \widetilde{r}\right)$ in the integrands of the expressions (35) and (36) is the spherical Hankel function of the first kind, $f_{l}\left(k_{3} \widetilde{r}\right) \equiv h_{l}\left(k_{3} \widetilde{r}\right)$, and $\widetilde{a}_{3, M}^{d}(l, m) \equiv \widetilde{a}_{3, M}^{d,>}(l, m) \quad$ and $\quad \widetilde{a}_{3, E}^{d}(l, m) \equiv \widetilde{a}_{3, E}^{d,>}(l, m)$ in the relationships (33) and (34). The function $f_{n}\left(k_{3} r\right)$ is the spherical Bessel function $j_{n}\left(k_{3} r\right)$ in the region $r<r_{d}$ and the spherical Hankel function $h_{n}\left(k_{3} r\right)$ if $r>r_{d}$. Assume first that dipole is located near the $\mathrm{O}_{2}$ center $\left(\widetilde{r}_{d} \approx 0\right)$. Then, $\quad f_{n}\left(k_{3} r\right) \equiv j_{n}\left(k_{3} r\right) \quad$ at $\quad r<D \quad$ and $f_{n}\left(k_{3} r\right) \equiv h_{n}\left(k_{3} r\right)$ at $r>D$. As an example, the results of numerical calculations of the linking coefficients $F_{n l}^{m}$ and $G_{n l}^{m}$ are shown in Figs 2 and 3 for the following set of parameters: $\{n, l, m\}=\{2,3,1\}, \varepsilon_{3}=3, \lambda_{0}=2 \pi / k_{0}=$ $500 \mathrm{~nm}, D=5 \mathrm{~nm}$. As seen from these figures, the coefficients $F_{n l}^{m}$ and $G_{n l}^{m}$ do not really depend on coordinate $r$, at which they are calculated (i.e. they can be calculated at an arbitrary $r$ ). At arbitrary finite $\widetilde{r}_{d}$, the parts of the dependences in these figures that get into the region $D-\widetilde{r}_{d}<r<D+\widetilde{r}_{d}$ should be simply deleted $\left(\tilde{r}<\widetilde{r}_{d}\right.$ in this region, therefore the function $f_{l}\left(k_{3} \widetilde{r}\right)$ in the integrands (35) and (36) should be the spherical Bessel function $j_{l}\left(k_{3} \widetilde{r}\right)$, the multipole coefficients $\widetilde{a}_{3, M}^{d}(l, m)$ and $\widetilde{a}_{3, E}^{d}(l, m)$ in (33) and (34) should be $\widetilde{a}_{3, M}^{d,<}(l, m)$ and $\widetilde{a}_{3, E}^{d,<}(l, m)$, respectively).

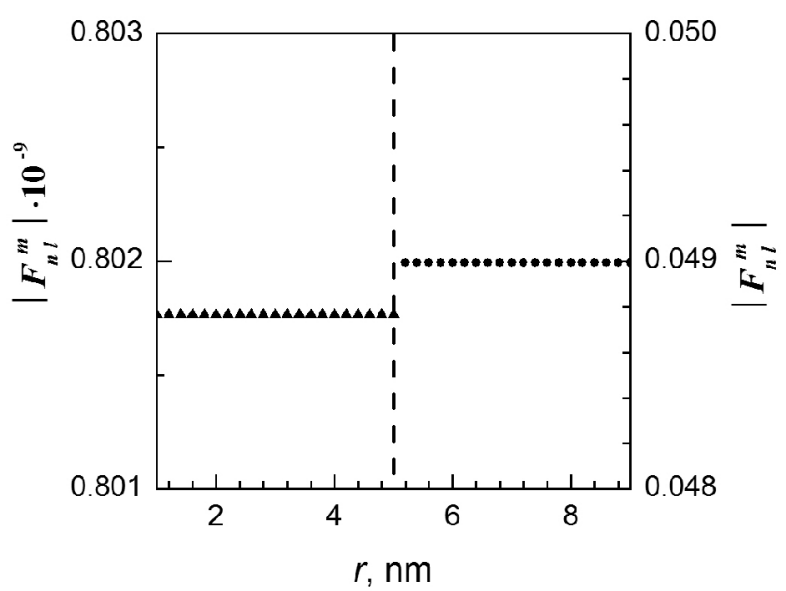

Fig. 2. Linking coefficient $\left|F_{n l}^{m}\right|$ as a function of $r$ in the case of $n=2, l=3, m=1, D=5 \mathrm{~nm}$. In the region $r<D$ $f_{n}\left(k_{3} r\right) \equiv j_{n}\left(k_{3} r\right), \quad\left|F_{n l}^{m}\right|$ values refer to the left axis of ordinates. In the region $r>D f_{n}\left(k_{3} r\right) \equiv h_{n}\left(k_{3} r\right),\left|F_{n l}^{m}\right|$ values refer to the right axis of ordinates.

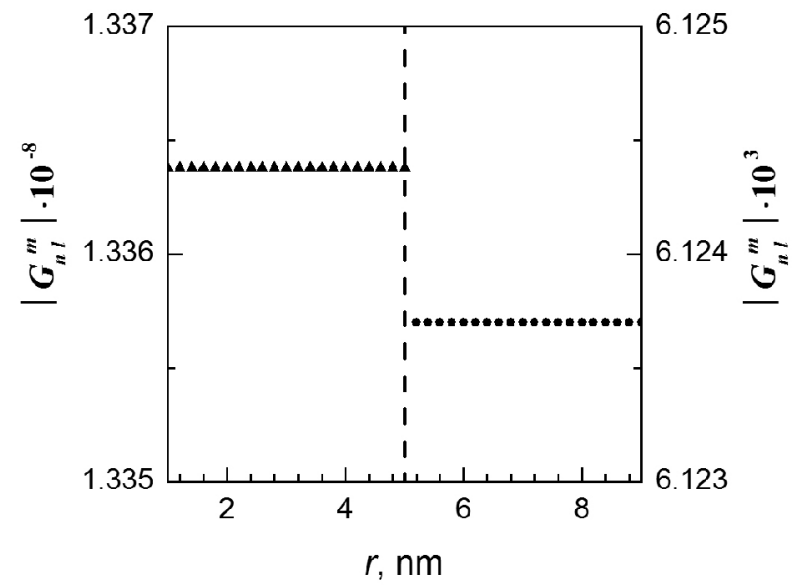

Fig. 3. Linking coefficient $\left|G_{n l}^{m}\right|$ as a function of $r$ in the case of $n=2, l=3, m=1, D=5 \mathrm{~nm}$. In the region $r<D$ $f_{n}\left(k_{3} r\right) \equiv j_{n}\left(k_{3} r\right),\left|G_{n l}^{m}\right|$ values refer to the left axis of ordinates. In the region $r>D f_{n}\left(k_{3} r\right) \equiv h_{n}\left(k_{3} r\right),\left|G_{n l}^{m}\right|$ values refer to the right axis of ordinates.

It can be shown that $F_{n l}^{m}=(-1)^{n+1} F_{l n}^{m} \quad$ and $G_{n l}^{m}=(-1)^{n+1} G_{l n}^{m}$.

If integrands in (35) and (36) expand in the Taylor series in powers of the distance $D$ between the two coordinate system origins, then instead of determining $F_{n l}^{m}$ and $G_{n l}^{m}$ by numerical integration, it becomes possible to find these coefficients analytically (see Appendix). However, in this case, an important note should be made. Taylor series contains the function $f_{l}\left(k_{3} \tilde{r}\right)$ and its derivatives that should be calculated at $D=0$. If $D$ tends to zero, then $\widetilde{r} \rightarrow r$ and $\widetilde{r}_{d} \rightarrow r_{d}$. For this reason, when considering the region $r<r_{d}$, the original region $\tilde{r}>\widetilde{r}_{d}$ at $D \neq 0$ transforms into the region $\tilde{r}<\tilde{r}_{d}$ at $D=0$. Correspondingly, the original function $f_{l}\left(k_{3} \tilde{r}\right)=h_{l}\left(k_{3} \tilde{r}\right)$ in the integrands (35) and (36), and the multipole coefficients $\tilde{a}_{3, M(E)}^{d}(l, m)=\widetilde{a}_{3, M(E)}^{d,>}(l, m)$ in the relationships (33) and (34) transform into $f_{l}\left(k_{3} \tilde{r}\right)=j_{l}\left(k_{3} \widetilde{r}\right) \quad$ and $\tilde{a}_{3, M(E)}^{d}(l, m)=\widetilde{a}_{3, M(E)}^{d,<}(l, m)$ due to the above outlined correlations (25) and (26). No such transformations occur when considering the region $r>r_{d}$. In this region, the original function $f_{l}\left(k_{3} \tilde{r}\right)=h_{l}\left(k_{3} \widetilde{r}\right)$ and the coefficients $\quad \widetilde{a}_{3, M(E)}^{d}(l, m)=\widetilde{a}_{3, M(E)}^{d,>}(l, m) \quad$ remain unchanged at $D \rightarrow 0$.

\section{B. Semiconductor QD in homogeneous medium}

Gradually approaching the QD+NP system in barrier matrix, consider a case of light emission by a point dipole located inside QD, when metal NP is absent in the 
system, i.e. when $\varepsilon_{1}=\varepsilon_{3} \neq \varepsilon_{2} \quad$ (see Fig. 1). The background permittivity $\varepsilon_{2}$ usually takes values within the range 3 to 15 . For instance, in CdTe static $\varepsilon_{2} \approx 10.4$, while in the optical frequency range $\varepsilon_{2} \approx 13$ [15]. EM field propagating from the point dipole to the QD-matrix boundary (i.e. incident $(i)$ on the inner QD boundary field) $\mathbf{E}_{2}^{i}(\widetilde{\mathbf{r}})$ and $\mathbf{B}_{2}^{i}(\widetilde{\mathbf{r}})$, as well as reflected $(r)$ inside the QD field $\mathbf{E}_{2}^{r}(\widetilde{\mathbf{r}})$ and $\mathbf{B}_{2}^{r}(\widetilde{\mathbf{r}})$ and transmitted $(t)$ into the matrix field $\mathbf{E}_{3}^{t}(\widetilde{\mathbf{r}})$ and $\mathbf{B}_{3}^{t}(\widetilde{\mathbf{r}})$ can be written in the standard forms (12) and (13) with the corresponding spherical Bessel or Hankel functions $f_{l}\left(k_{n} \tilde{r}\right)$, depending on the necessity to provide the field finiteness at $\tilde{r}=0$ or right field asymptotic behavior at $\widetilde{r} \rightarrow \infty$. For short, only electric fields are written below in order to make the introduced notations clear:

$$
\begin{aligned}
& \mathbf{E}_{2}^{i}(\widetilde{\mathbf{r}})=\sum_{l, m}\left\{i /\left(k_{0} \varepsilon_{2}\right) \tilde{a}_{2, E}^{i}(l, m)\left[\nabla_{\widetilde{\mathbf{r}}} \times\right.\right. \\
& \left.\left.\times h_{l}\left(k_{2} \widetilde{r}\right) \mathbf{X}_{l m}(\widetilde{\Omega})\right]+\widetilde{a}_{2, M}^{i}(l, m) h_{l}\left(k_{2} \widetilde{r}\right) \mathbf{X}_{l m}(\widetilde{\Omega})\right\}, \\
& \mathbf{E}_{2}^{r}(\widetilde{\mathbf{r}})=\sum_{l, m}\left\{i /\left(k_{0} \varepsilon_{2}\right) \tilde{a}_{2, E}^{r}(l, m)\left[\nabla_{\widetilde{\mathbf{r}}} \times\right.\right. \\
& \left.\left.\times j_{l}\left(k_{2} \widetilde{r}\right) \mathbf{X}_{l m}(\widetilde{\Omega})\right]+\widetilde{a}_{2, M}^{r}(l, m) j_{l}\left(k_{2} \tilde{r}\right) \mathbf{X}_{l m}(\tilde{\Omega})\right\}, \\
& \mathbf{E}_{3}^{t}(\widetilde{\mathbf{r}})=\sum_{l, m}\left\{i /\left(k_{0} \varepsilon_{3}\right) \widetilde{a}_{3, E}^{t}(l, m)\left[\nabla_{\widetilde{\mathbf{r}}} \times\right.\right. \\
& \left.\left.\times h_{l}\left(k_{3} \tilde{r}\right) \mathbf{X}_{l m}(\widetilde{\Omega})\right]+\widetilde{a}_{3, M}^{t}(l, m) h_{l}\left(k_{3} \tilde{r}\right) \mathbf{X}_{l m}(\widetilde{\Omega})\right\}
\end{aligned}
$$

The multipole coefficients $\tilde{a}_{2, E}^{i}(l, m)$ and $\tilde{a}_{2, M}^{i}(l, m)$ are the coefficients $\tilde{a}_{2, E}^{d,>}(l, m)$ (27) and $\tilde{a}_{2, M}^{d,>}(l, m)$ (29), correspondingly (tilde means that they are written in a coordinate system with the center in QD).

From this point, all further consideration is valid as well in the case of light emission by QD as a whole. The only difference from the case of light emission by the point dipole located inside QD is that the QD multipole amplitudes $\widetilde{a}_{Q D, E}^{i}(l, m)$ and $\tilde{a}_{Q D, M}^{i}(l, m)$ should be used in (40) as $\tilde{a}_{2, E}^{i}(l, m)$ and $\tilde{a}_{2, M}^{i}(l, m)$ instead of point dipole multipole amplitudes $\widetilde{a}_{2, E}^{d,>}(l, m)$ and $\widetilde{a}_{2, M}^{d,>}(l, m)$, correspondingly. As it was already noted, the amplitudes $\tilde{a}_{Q D, E}^{i}(l, m)$ and $\tilde{a}_{Q D, M}^{i}(l, m)$ can be found by integrating contributions of all the point dipoles (unit cells) inside QD, the contributions being determined by the exciton wave function. The lowest in energy quantized exciton state is fivefold degenerate in $F_{z^{-}}$ projections of the total electron-hole angular momentum $F=2$ in the case of QD material of cubic modification with the fourfold valence band $\Gamma_{8}[16]$. To avoid a significant increase in the present work volume, the calculation of the amplitudes $\tilde{a}_{Q D, E}^{i}(l, m)$ and
$\widetilde{a}_{Q D, M}^{i}(l, m)$ on the base of the corresponding exciton wave functions as well as the calculation of light emission characteristics of the whole QD+NP system will be presented in a separate work. In brief, the main result of this consideration is that the EM field radiated by QD as a whole contains only dipole $(l=1)$ and octupole $(l=3) \quad$ electric-type components and quadrupole $(l=2)$ magnetic-type components, all these components of EM field arising due to the presence of non-spherical parts in the exciton wave function (i.e. due to the spin-orbit interaction and mixing of the heavy and light hole states in QD).

From the continuity conditions for the tangential components of the electric and magnetic fields at the QD surface, two systems of algebraic equations for the multipole coefficients can be obtained in the standard way [12]. As follows from these equations, $\tilde{a}_{3, M}^{t}(l, m)=V_{M, 23}^{Q D}(l) \widetilde{a}_{2, M}^{i}(l, m)$ and $\tilde{a}_{3, E}^{t}(l, m)=V_{E, 23}^{Q D}(l) \tilde{a}_{2, E}^{i}(l, m)$. Hereafter, the indices $j$ and $k$ in the transmission and reflection amplitudes $V_{M(E), j k}^{Q D(N)}$ are used to indicate the medium from which the field is incident on the boundary (first index $j$ ) and the medium to which the field is transferred (second index $k$ ). Taking into account that Wronskian $\mathrm{W}\left[j_{l}(x), h_{l}(x)\right]=i / x^{2}$, the following expressions for $V_{M, 23}^{Q D}(l)$ and $V_{E, 23}^{Q D}(l)$ can be obtained:

$V_{M, 23}^{Q D}(l)=\frac{i \sqrt{\varepsilon_{2}}}{\left(k_{2} R_{2}\right)^{2}} \frac{1}{\sqrt{\varepsilon_{3}} j_{l}\left(k_{2} R_{2}\right) h_{l}^{\prime}\left(k_{3} R_{2}\right)-\sqrt{\varepsilon_{2}} h_{l}\left(k_{3} R_{2}\right) j_{l}^{\prime}\left(k_{2} R_{2}\right)}$,

$V_{E, 23}^{Q D}(l)=$

$=\frac{i \varepsilon_{3}}{\left(k_{2} R_{2}\right)} \frac{1}{\varepsilon_{2} j_{l}\left(k_{2} R_{2}\right)\left[x h_{l}(x)\right]_{x=k_{3} R_{2}}^{\prime}-\varepsilon_{3} h_{l}\left(k_{3} R_{2}\right)\left[x j_{l}(x)\right]_{x=k_{2} R_{2}}^{\prime}}$

Products $k_{2} R_{2}$ and $k_{3} R_{2}$ take values within the range $0.01 \ldots 0.2$ for the QDs with the radius $R_{2}-1 \ldots 5 \mathrm{~nm}, \varepsilon_{2}$ and $\varepsilon_{3}$ values $-1 \ldots 15$ and the wavelength $\lambda_{0}-$ $400 \ldots 800 \mathrm{~nm}$. Thus, these products are small enough to use limit values $j_{l}(x) \approx x^{l} /(2 l+1) !$ and $h_{l}(x) \approx-i(2 l-1) ! ! / x^{l+1}$ of the spherical Bessel and Hankel functions at small arguments. As a result, the following approximate expressions for $V_{M, 23}^{Q D}(l)$ and $V_{E, 23}^{Q D}(l)$ can be obtained from (43) and (44):

$$
\begin{aligned}
& V_{M, 23}^{Q D}(l) \approx\left(\varepsilon_{3} / \varepsilon_{2}\right)^{(l+1) / 2}, \\
& V_{E, 23}^{Q D}(l) \approx\left(\varepsilon_{3} / \varepsilon_{2}\right)^{(l+1) / 2}(2 l+1) /\left[l \varepsilon_{2} / \varepsilon_{3}+(l+1)\right] .
\end{aligned}
$$

As seen from (45) and (46), the transmission amplitudes $V_{M, 23}^{Q D}(l)$ and $V_{E, 23}^{Q D}(l)$ are the functions of 
the multipole number $l$, and for this reason, the field emitted by QD cannot be represented as that of point dipole in the case of light emission by the point dipole located inside QD, not to mention the case of light emission by QD as a whole, when only components with $l=1,2$ and 3 form the radiated EM field.

Denote by index $i$ the above transmitted EM fields $\mathbf{E}_{3}^{t}(\tilde{\mathbf{r}})$ and $\mathbf{B}_{3}^{t}(\tilde{\mathbf{r}})$ expressed in spherical coordinate system with its origin at the point $\mathrm{O}_{1}$ (see figure 1); the corresponding fields will be $\mathbf{E}_{3, N P}^{i}(\mathbf{r})$ and $\mathbf{B}_{3, N P}^{i}(\mathbf{r})$. These fields can be written in standard forms (12) and (13) with the multipole coefficients $a_{n, E}(l, m)=a_{3, E}^{i}(l, m), \quad a_{n, M}(l, m)=a_{3, M}^{i}(l, m) \quad$ and $f_{l}\left(k_{3} r\right) \equiv j_{l}\left(k_{3} r\right) \quad$ (the spherical Bessel function $j_{l}\left(k_{3} r\right)$ provides the field finiteness at $\left.r=0\right)$. The relationships between the multipole coefficients $a_{3, M}^{i}$ and $a_{3, E}^{i}$ of this field and the multipole coefficients $\tilde{a}_{3, M}^{t}$ and $\tilde{a}_{3, E}^{t}$ of the same field written in the coordinate system with its origin at the point $\mathrm{O}_{2}$ are quite analogous to the relationships (33) and (34) in the case of completely homogeneous medium:

$$
\begin{aligned}
& a_{3, M}^{i}(n, m)=\sum_{l=1}^{\infty}\left[F_{n l}^{m}(D) \widetilde{a}_{3, M}^{t}(l, m)+\right. \\
& \left.+m\left(k_{3} D / \sqrt{\varepsilon_{3}}\right) G_{n l}^{m}(D) \widetilde{a}_{3, E}^{t}(l, m)\right], \\
& a_{3, E}^{i}(n, m)=\sum_{l=1}^{\infty}\left[F_{n l}^{m}(D) \widetilde{a}_{3, E}^{t}(l, m)-\right. \\
& \left.-m\left(k_{3} D\right) \sqrt{\varepsilon_{3}} G_{n l}^{m}(D) \widetilde{a}_{3, M}^{t}(l, m)\right] .
\end{aligned}
$$

\section{Interaction of electromagnetic field emitted by QD with metal NP}

By determining the multipole coefficients $a_{3, M}^{i}(n, m)$ and $a_{3, E}^{i}(n, m)$ of the field radiated by QD in the coordinate system with its origin at the point $\mathrm{O}_{1}$, we have defined thus the incident $(i)$ field on spherical metal NP if NP is additionally placed into the system. Now, it becomes possible to consider the Mie scattering by NP, i.e. to find reflected $(r)$ from NP and transmitted $(t)$ into NP fields (nonradiative losses due to electron scattering inside NP are related only with these transmitted fields).

Scattered (reflected) fields $\mathbf{E}_{3, N P}^{r}(\mathbf{r})$ and $\mathbf{B}_{3, N P}^{r}(\mathbf{r})$ can be written in the standard forms (12) and (13) with the multipole coefficients $a_{n, E}(l, m)=a_{3, E}^{r}(l, m)$, $a_{n, M}(l, m)=a_{3, M}^{r}(l, m) \quad$ and $\quad f_{l}\left(k_{3} r\right) \equiv h_{l}\left(k_{3} r\right) \quad$ (the spherical Hankel function $h_{l}\left(k_{3} r\right)$ of the first kind provides correct asymptotic behavior of the scattered EM field in the form of diverging spherical waves at $r \rightarrow \infty)$.
With account of spatial dispersion in the NP permittivity (10) the electric field induced in (transmitted into) the metal NP should be a sum of the transverse and longitudinal fields, $\mathbf{E}_{1}^{t}(\mathbf{r})=\mathbf{E}_{1, T}^{t}(\mathbf{r})+\mathbf{E}_{1, L}^{t}(\mathbf{r})$, where the transverse electric field can be expressed as

$\mathbf{E}_{1, T}^{t}(\mathbf{r})=\sum_{l, m}\left\{i /\left[k_{0} \varepsilon_{1}^{T}(\omega, 0)\right] a_{1, E}^{t}(l, m) \times\right.$

$\left.\times\left[\nabla \times j_{l}\left(k_{T} r\right) \mathbf{X}_{l m}(\Omega)\right]+a_{1, M}^{t}(l, m) j_{l}\left(k_{T} r\right) \mathbf{X}_{l m}(\Omega)\right\}$,

and the longitudinal electric field as

$\mathbf{E}_{1, L}^{t}(\mathbf{r})=\sum_{l, m} a_{1, L}^{t}(l, m)\left(1 / k_{L}\right) \nabla\left[j_{l}\left(k_{L} r\right) Y_{l m}(\Omega)\right]$.

Correspondingly, the magnetic field inside the metal NP can be expressed as

$\mathbf{B}_{1}^{t}(\mathbf{r})=\sum_{l, m}\left\{a_{1, E}^{t}(l, m) j_{l}\left(k_{T} r\right) \mathbf{X}_{l m}(\Omega)-\right.$

$\left.-\left(i / k_{0}\right) a_{1, M}^{t}(l, m)\left[\nabla \times j_{l}\left(k_{T} r\right) \mathbf{X}_{l m}(\Omega)\right]\right\}$.

From the continuity conditions for the tangential components of the electric and magnetic fields and the additional boundary condition (ABC) of turning to zero of the normal component of free carrier's current density at the NP surface $\left.\mathbf{j} \cdot \mathbf{e}_{r}\right|_{r=R_{1}}=0$, it can be found that $a_{3, M}^{r}(l, m)=V_{M, 33}^{N P}(l) a_{3, M}^{i}(l, m), a_{1, M}^{t}(l, m)=V_{M, 31}^{N P}(l) a_{3, M}^{i}(l, m)$, $a_{3, E}^{r}(l, m)=V_{E, 33}^{N P}(l) a_{3, E}^{i}(l, m), a_{1, E}^{t}(l, m)=V_{E, 31}^{N P}(l) a_{3, E}^{i}(l, m)$ and $\quad a_{1, L}^{t}(l, m)=V_{L, 31}^{N P}(l) a_{3, E}^{i}(l, m), \quad$ where the transmission and reflection amplitudes are expressed as $V_{M, 33}^{N P}(l)=$

$=-\frac{\left[\sqrt{\varepsilon_{1}^{T}(\omega, 0)} j_{l}\left(k_{3} R_{1}\right) j_{l}^{\prime}\left(k_{T} R_{1}\right)-\sqrt{\varepsilon_{3}} j_{l}\left(k_{T} R_{1}\right) j_{l}^{\prime}\left(k_{3} R_{1}\right)\right]}{\Delta_{M}}$,

$V_{M, 31}^{N P}(l)=-i \sqrt{\varepsilon_{3}} /\left[\left(k_{3} R_{1}\right)^{2} \Delta_{M}\right]$,

$V_{E, 33}^{N P}(l)=-\left\{\varepsilon_{1}^{T}(\omega, 0) j_{l}\left(k_{T} R_{1}\right)\left[x j_{l}(x)\right]_{x=k_{3} R_{1}}^{\prime}-\right.$

$\left.-\varepsilon_{3} j_{l}\left(k_{3} R_{1}\right)\left[x j_{l}(x)\right]_{x=k_{T} R_{1}}^{\prime}-\left(C \varepsilon_{1}^{T}(\omega, 0)-\varepsilon_{3}\right) A\right\} / \Delta_{E L}$,

$V_{E, 31}^{N P}(l)=i \varepsilon_{1}^{T}(\omega, 0) /\left(k_{3} R_{1} \Delta_{E L}\right)$,

$V_{L, 31}^{N P}(l)=\frac{-i \sqrt{l(l+1)}\left[C \varepsilon_{1}^{T}(\omega, 0)-\varepsilon_{3}\right] j_{l}\left(k_{T} R_{1}\right)}{\left[\varepsilon_{3} k_{3} k_{0} R_{1}^{2} j_{l}^{\prime}\left(k_{L} R_{1}\right) \Delta_{E L}\right]}$,

$\Delta_{M}=\sqrt{\varepsilon_{1}^{T}(\omega, 0)} h_{l}\left(k_{3} R_{1}\right) j_{l}^{\prime}\left(k_{T} R_{1}\right)-$

$-\sqrt{\varepsilon_{3}} j_{l}\left(k_{T} R_{1}\right) h_{l}^{\prime}\left(k_{3} R_{1}\right)$,

$\Delta_{E L}=\varepsilon_{1}^{T}(\omega, 0) j_{l}\left(k_{T} R_{1}\right)\left[x h_{l}(x)\right]_{x=k_{3} R_{1}}^{\prime}-$

$-\varepsilon_{3} h_{l}\left(k_{3} R_{1}\right)\left[x j_{l}(x)\right]_{x=k_{T} R_{1}}^{\prime}-\left(C \varepsilon_{1}^{T}(\omega, 0)-\varepsilon_{3}\right) B$,

$A=\frac{l(l+1) j_{l}\left(k_{L} R_{1}\right) j_{l}\left(k_{T} R_{1}\right) j_{l}\left(k_{3} R_{1}\right)}{k_{L} R_{1} j_{l}^{\prime}\left(k_{L} R_{1}\right)}$,

(C) 2013, V. Lashkaryov Institute of Semiconductor Physics, National Academy of Sciences of Ukraine 


$$
\begin{aligned}
& B=\frac{l(l+1) j_{l}\left(k_{L} R_{1}\right) j_{l}\left(k_{T} R_{1}\right) h_{l}\left(k_{3} R_{1}\right)}{k_{L} R_{1} j_{l}^{\prime}\left(k_{L} R_{1}\right)}, \\
& C=\varepsilon_{3} /\left[1+\varepsilon_{i b}(\omega)\right] .
\end{aligned}
$$

The poles in the amplitudes $V_{E, 33}^{N P}(l), V_{E, 31}^{N P}(l)$ and $V_{L, 31}^{N P}(l)$ (zeros of $\Delta_{E L}$ ) correspond in energies to the localized surface plasmon resonances of the corresponding order $l$. Thus, at resonant excitation of NP (or close to resonant, depending on the frequency of light emitted by QD), just these localized plasmons substantially influence the characteristic radiative and non-radiative lifetimes in the QD+NP system.

Using the relationship $\mathbf{j}_{T(L)}=-i \omega \mathbf{P}_{p l}^{T(L)}$ between free carrier's current densities $\mathbf{j}_{T(L)}$ and corresponding polarizations $\mathbf{P}_{p l}^{T(L)}$, the ABC $\left.\mathbf{j} \cdot \mathbf{e}_{r}\right|_{r=R_{1}}=\left.\left(\mathbf{j}_{T}+\mathbf{j}_{L}\right) \cdot \mathbf{e}_{r}\right|_{r=R_{1}}=0$ can be rewritten as $\left.\left(\mathbf{P}_{p l}^{T}+\mathbf{P}_{p l}^{L}\right) \cdot \mathbf{e}_{r}\right|_{r=R_{1}}=$ $=\left.\left(\varepsilon_{p l}^{T}(\omega, 0) \mathbf{E}_{1, T}^{t}+\varepsilon_{p l}^{L}\left(\omega, k_{L}\right) \mathbf{E}_{1, L}^{t}\right) \cdot \mathbf{e}_{r}\right|_{r=R_{1}}=0 . \quad$ Further, taking into account that $\varepsilon_{p l}^{L}\left(\omega, k_{L}\right) \mathbf{E}_{1, L}^{t}=-\left[1+\varepsilon_{i b}(\omega)\right] \mathbf{E}_{1, L}^{t}$ and $\left.\quad \varepsilon_{p l}^{T}(\omega, 0) \mathbf{E}_{1, T}^{t} \cdot \mathbf{e}_{r}\right|_{r=R_{1}}=$ $=\left.\left[\varepsilon_{3}\left(\mathbf{E}_{3, N P}^{i}+\mathbf{E}_{3, N P}^{r}\right)-\left(1+\varepsilon_{i b}(\omega)\right) \mathbf{E}_{1, T}^{t}\right] \cdot \mathbf{e}_{r}\right|_{r=R_{1}}, \quad$ the $\left.\mathrm{ABC} \mathbf{j} \cdot \mathbf{e}_{r}\right|_{r=R_{1}}$ can be finally reduced to the boundary condition $\left.\quad\left[1+\varepsilon_{i b}(\omega)\right]\left(\mathbf{E}_{1, T}^{t}+\mathbf{E}_{1, L}^{t}\right) \cdot \mathbf{e}_{r}\right|_{r=R_{1}}=$ $=\left.\varepsilon_{3}\left(\mathbf{E}_{3, N P}^{i}+\mathbf{E}_{3, N P}^{r}\right) \cdot \mathbf{e}_{r}\right|_{r=R_{1}}$. It transforms into the conventional $\mathrm{ABC}$ of the continuity of the normal component of the electric field at the NP surface [11] only in the case of simple metal NP $\left(\varepsilon_{i b} \equiv 0\right)$ in vacuum $\left(\varepsilon_{3}=1\right)$, or, more generally, when interband contribution $\varepsilon_{i b}$ to the NP permittivity can be considered as a constant and $1+\varepsilon_{i b}=\varepsilon_{\infty}=\varepsilon_{3}$. The modernized $\mathrm{ABC}$ used in this work was first introduced in the work [17]. This $A B C$ is a generalization of a conventional $\mathrm{ABC}$ for the case of real metal $\mathrm{NP}$ in dielectric matrix.

In the long-wave limit when $k_{3} R_{1}$, $\left|k_{T} R_{1}\right|,\left|k_{L} R_{1}\right|<<1$, the following approximate expressions can be obtained for the amplitudes (52)(56):

$$
\begin{aligned}
& V_{M, 33}^{N P}(l) \approx i \frac{\left(k_{3} R_{1}\right)^{2 l+3}}{(2 l+3) ! !(2 l+1) ! !} \frac{\varepsilon_{1}^{T}(\omega, 0)-\varepsilon_{3}}{\varepsilon_{3}}, \\
& V_{M, 31}^{N P}(l) \approx\left(\frac{\varepsilon_{3}}{\varepsilon_{1}^{T}(\omega, 0)}\right)^{l / 2}, \\
& V_{E, 33}^{N P}(l) \approx i \frac{\left(k_{3} R_{1}\right)^{2 l+1}}{(2 l+1) ! !(2 l-1) ! !} \frac{(l+1)(1-C)}{C(l+1)+l},
\end{aligned}
$$

$$
\begin{aligned}
& V_{E, 31}^{N P}(l) \approx\left(\frac{\varepsilon_{3}}{\varepsilon_{1}^{T}(\omega, 0)}\right)^{l / 2} \frac{2 l+1}{C(l+1)+l}, \\
& V_{L, 31}^{N P}(l) \approx \frac{2 l+1}{C(l+1)+l} \times \\
& \times \sqrt{\frac{l+1}{l}}\left(k_{0} / k_{L}\right)^{l-1} \varepsilon_{3}^{l / 2-1}\left(\frac{\varepsilon_{3}}{\varepsilon_{1}^{T}(\omega, 0)}-C\right),
\end{aligned}
$$

By determining multipole coefficients $a_{3, M}^{r}(l, m)$, $a_{3, E}^{r}(l, m), \quad a_{1, E}^{t}(l, m), \quad a_{1, M}^{t}(l, m)$ and $a_{1, L}^{t}(l, m)$, we have determined thus all the EM fields in the nonspherical "semiconductor QD + metal NP" system in the single scattering approximation.

\section{Light emission by QD + NP system with account of multiple scattering of electromagnetic field}

To complete the consideration, the multiple scattering between QD and NP of the EM field emitted initially by QD should be taken into account. In this case, the reflected from NP field $\mathbf{E}_{3, N P}^{r}(\mathbf{r})$ and $\mathbf{B}_{3, N P}^{r}(\mathbf{r})$, in turn, should be considered as incident on QD. In the spherical coordinate system with its origin in QD, this field should have the following form:

$\mathbf{E}_{3, Q D}^{i}(\widetilde{\mathbf{r}})=\sum_{l, m}\left\{i /\left(k_{0} \varepsilon_{3}\right) \widetilde{a}_{3, E}^{i}(l, m)\left[\nabla_{\widetilde{\mathbf{r}}} \times\right.\right.$

$\left.\left.\times j_{l}\left(k_{3} \widetilde{r}\right) \mathbf{X}_{l m}(\widetilde{\Omega})\right]+\widetilde{a}_{3, M}^{i}(l, m) j_{l}\left(k_{3} \widetilde{r}\right) \mathbf{X}_{l m}(\widetilde{\Omega})\right\}$,

$\mathbf{B}_{3, Q D}^{i}(\widetilde{\mathbf{r}})=\sum_{l, m}\left\{\tilde{a}_{3, E}^{i}(l, m) j_{l}\left(k_{3} \widetilde{r}\right) \mathbf{X}_{l m}(\widetilde{\Omega})_{-}\right.$

$\left.-\left(i / k_{0}\right) \widetilde{a}_{3, M}^{i}(l, m)\left[\nabla_{\widetilde{\mathbf{r}}} \times j_{l}\left(k_{3} \widetilde{r}\right) \mathbf{X}_{l m}(\widetilde{\Omega})\right]\right\}$.

From the identities $\mathbf{E}_{3, Q D}^{i}(\widetilde{\mathbf{r}}) \equiv \mathbf{E}_{3, N P}^{r}(\mathbf{r})$ and $\mathbf{B}_{3, Q D}^{i}(\widetilde{\mathbf{r}}) \equiv \mathbf{B}_{3, N P}^{r}(\mathbf{r})$, the relationships can be obtained between the coefficients $\widetilde{a}_{3, M}^{i}$ and $\widetilde{a}_{3, E}^{i}$ on the one hand, and $a_{3, M}^{r}$ and $a_{3, E}^{r}$ on the other hand. The relationships turned out to be similar to (47) and (48):

$\tilde{a}_{3, M}^{i}(n, m)=\sum_{l=1}^{\infty}(-1)^{l+n}\left[F_{n l}^{m}(D) a_{3, M}^{r}(l, m)-\right.$

$\left.-m\left(k_{3} D / \sqrt{\varepsilon_{3}}\right) G_{n l}^{m}(D) a_{3, E}^{r}(l, m)\right]$,

$\widetilde{a}_{3, E}^{i}(n, m)=\sum_{l=1}^{\infty}(-1)^{l+n}\left[F_{n l}^{m}(D) a_{3, E}^{r}(l, m)+\right.$

$\left.+m\left(k_{3} D\right) \sqrt{\varepsilon_{3}} G_{n l}^{m}(D) a_{3, M}^{r}(l, m)\right]$.

With the multipole coefficients $\tilde{a}_{3, M}^{i}$ and $\widetilde{a}_{3, E}^{i}$ found in this way, the standard Mie scattering of the EM field by QD can be further considered. According to (12), the reflected electric field from QD should have the following form: 


$$
\begin{aligned}
& \mathbf{E}_{3, Q D}^{r}(\widetilde{\mathbf{r}})=\sum_{l, m}\left\{i /\left(k_{0} \varepsilon_{3}\right) \widetilde{a}_{3, E}^{r}(l, m)\left[\nabla_{\widetilde{\mathbf{r}}} \times h_{l}\left(k_{3} \widetilde{r}\right) \times\right.\right. \\
& \left.\left.\times \mathbf{X}_{l m}(\widetilde{\Omega})\right]+\widetilde{a}_{3, M}^{r}(l, m) h_{l}\left(k_{3} \widetilde{r}\right) \mathbf{X}_{l m}(\widetilde{\Omega})\right\} .
\end{aligned}
$$

The multipole coefficients of the reflected field are related with the corresponding coefficients of the incident field by the Mie scattering amplitudes $V_{M, 33}^{Q D}(l)$ and $\quad V_{E, 33}^{Q D}(l): \quad \tilde{a}_{3, M}^{r}(l, m)=V_{M, 33}^{Q D}(l) \widetilde{a}_{3, M}^{i}(l, m) \quad$ and $\tilde{a}_{3, E}^{r}(l, m)=V_{E, 33}^{Q D}(l) \tilde{a}_{3, E}^{i}(l, m)$. These amplitudes are expressed as follows:

$V_{M, 33}^{Q D}(l)=-\frac{\sqrt{\varepsilon_{2}} j_{l}\left(k_{3} R_{2}\right) j_{l}^{\prime}\left(k_{2} R_{2}\right)-\sqrt{\varepsilon_{3}} j_{l}\left(k_{2} R_{2}\right) j_{l}^{\prime}\left(k_{3} R_{2}\right)}{\sqrt{\varepsilon_{2}} h_{l}\left(k_{3} R_{2}\right) j_{l}^{\prime}\left(k_{2} R_{2}\right)-\sqrt{\varepsilon_{3}} j_{l}\left(k_{2} R_{2}\right) h_{l}^{\prime}\left(k_{3} R_{2}\right)}$,

$V_{E, 33}^{Q D}(l)=-\left\{j_{l}\left(k_{3} R_{2}\right)\left[j_{l}\left(k_{2} R_{2}\right)\left(\varepsilon_{3} / \varepsilon_{2}-1\right)+\right.\right.$

$\left.\left.+k_{2} R_{2} j_{l}^{\prime}\left(k_{2} R_{2}\right) \varepsilon_{3} / \varepsilon_{2}\right]-k_{3} R_{2} j_{l}\left(k_{2} R_{2}\right) j_{l}^{\prime}\left(k_{3} R_{2}\right)\right\} /$

$/\left\{h_{l}\left(k_{3} R_{2}\right)\left[j_{l}\left(k_{2} R_{2}\right)\left(\varepsilon_{3} / \varepsilon_{2}-1\right)+k_{2} R_{2} j_{l}^{\prime}\left(k_{2} R_{2}\right) \varepsilon_{3} / \varepsilon_{2}\right]-\right.$ $\left.-k_{3} R_{2} j_{l}\left(k_{2} R_{2}\right) h_{l}^{\prime}\left(k_{3} R_{2}\right)\right\}$.

In the long-wave limit, when $k_{3} R_{2}, k_{2} R_{2}<<1$

$V_{M, 33}^{Q D}(l) \approx i \frac{\left(k_{3} R_{2}\right)^{2 l+3}}{(2 l+3) ! !(2 l+1) ! !} \frac{\varepsilon_{2}-\varepsilon_{3}}{\varepsilon_{3}}$,

$V_{E, 33}^{Q D}(l) \approx i \frac{\left(k_{3} R_{2}\right)^{2 l+1}}{(2 l+1) !(2 l-1) ! !} \frac{(l+1)\left(\varepsilon_{2}-\varepsilon_{3}\right)}{\varepsilon_{3}(l+1)+\varepsilon_{2} l}$.

Reflected from QD field, in turn, gives additional contributions to the total radiated far-field and to the fields reflected from and transmitted into the NP. Being repeated, this process of mutual scatterings should form the series of corresponding contributions to the total fields. The resulting total fields should have the following forms (for short, only electric fields are written below):

$\mathbf{E}_{3}^{Q D}(\widetilde{\mathbf{r}})=\sum_{l, m}\left\{i /\left(k_{0} \varepsilon_{3}\right) \tilde{A}_{3, E}(l, m)\left[\nabla_{\widetilde{\mathbf{r}}} \times h_{l}\left(k_{3} \widetilde{r}\right) \times\right.\right.$

$\left.\left.\times \mathbf{X}_{l m}(\widetilde{\Omega})\right]+\widetilde{A}_{3, M}(l, m) h_{l}\left(k_{3} \widetilde{r}\right) \mathbf{X}_{l m}(\widetilde{\Omega})\right\}$,

$\mathbf{E}_{3}^{N P}(\mathbf{r})=\sum_{l, m}\left\{i /\left(k_{0} \varepsilon_{3}\right) A_{3, E}(l, m)\left[\nabla \times h_{l}\left(k_{3} r\right) \mathbf{X}_{l m}(\Omega)\right]+\right.$

$\left.+A_{3, M}(l, m) h_{l}\left(k_{3} r\right) \mathbf{X}_{l m}(\Omega)\right\}$,

$\mathbf{E}_{1, T}^{N P}(\mathbf{r})=\sum_{l, m}\left\{i /\left[k_{0} \varepsilon_{1}^{T}(\omega, 0)\right] A_{1, E}(l, m) \times\right.$

$\left.\times\left[\nabla \times j_{l}\left(k_{T} r\right) \mathbf{X}_{l m}(\Omega)\right]+A_{1, M}(l, m) j_{l}\left(k_{T} r\right) \mathbf{X}_{l m}(\Omega)\right\}$,

$\mathbf{E}_{1, L}^{N P}(\mathbf{r})=\sum_{l, m} A_{1, L}(l, m)\left(1 / k_{L}\right) \nabla\left[j_{l}\left(k_{L} r\right) Y_{l m}(\Omega)\right]$.

The electric field $\mathbf{E}_{3}^{Q D}(\widetilde{\mathbf{r}})$ is the sum of the field (42), which is initially radiated by QD and thus can be considered as zero-order contribution, and all subsequently reflected from (scattered by) QD fields (higher order contributions). Analogously, the electric field $\mathbf{E}_{3}^{N P}(\mathbf{r})$ is the sum of all scattered by NP fields that are formed in the process of multiple scatterings in the $\mathrm{QD}+\mathrm{NP}$ system, $\mathbf{E}_{1, T}^{N P}(\mathbf{r})$ is the sum of all induced in NP transverse fields, and $\mathbf{E}_{1, L}^{N P}(\mathbf{r})$ is the sum of all induced in NP longitudinal fields. The corresponding total magnetic fields $\mathbf{B}_{3}^{Q D}(\widetilde{\mathbf{r}}), \mathbf{B}_{3}^{N P}(\mathbf{r})$ and $\mathbf{B}_{1}^{N P}(\mathbf{r})$ can be expressed analogously to the total electric fields (76)(78) using the general form of EM field presentation (13).

To continue further and find multipole coefficients $\widetilde{A}_{3, E}, \widetilde{A}_{3, M}, A_{3, E}, A_{3, M}, A_{1, E}, A_{1, M}$ and $A_{1, L}$ of the total fields (76)-(79), introduce the following columns of these coefficients:

$$
\begin{aligned}
& {\left[\begin{array}{lll}
\widetilde{A}_{3, m}
\end{array}\right]=\left[\begin{array}{lll}
\widetilde{A}_{3, M}(1, m) & \ldots & \widetilde{A}_{3, M}(l, m)
\end{array} \ldots\right.} \\
& \text {.. } \left.\tilde{A}_{3, E}(1, m) \quad \ldots \quad \widetilde{A}_{3, E}(l, m) \quad \ldots\right]^{T}, \\
& {\left[A_{3, m}\right]=\left[\begin{array}{lll}
A_{3, M}(1, m) & \ldots & A_{3, M}(l, m)
\end{array} \ldots\right.} \\
& \text {.. } \left.A_{3, E}(1, m) \quad \ldots \quad A_{3, E}(l, m) \quad \ldots\right]^{T}, \\
& {\left[A_{1, m}^{T}\right]=\left[A_{1, M}(1, m) \ldots A_{1, M}(l, m) \ldots A_{1, E}(1, m) \ldots\right.} \\
& \text {... } \left.A_{1, E}(l, m) \quad \ldots\right]^{T} \text {, } \\
& {\left[A_{1, m}^{L}\right]=\left[\begin{array}{llll}
A_{1, L}(1, m) & \ldots & A_{1, L}(l, m) & \ldots
\end{array}\right]^{T},}
\end{aligned}
$$

and rewrite all the above obtained linear relationships in a matrix form. Thus, the relationships $\widetilde{a}_{3, M}^{t}(l, m)=V_{M, 23}^{Q D}(l) \widetilde{a}_{2, M}^{i}(l, m) \quad$ and $\widetilde{a}_{3, E}^{t}(l, m)=V_{E, 23}^{Q D}(l) \widetilde{a}_{2, E}^{i}(l, m)$ at the QD boundary can be written as $\left[\widetilde{a}_{3, m}^{t}\right]=\left[V_{23}^{Q D}\right]\left[\widetilde{a}_{2, m}^{i}\right]$, where $\left[\widetilde{a}_{3, m}^{t}\right]$ and $\left[\widetilde{a}_{2, m}^{i}\right]$ are the columns

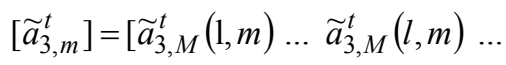

$\left.\ldots \widetilde{a}_{3, E}^{t}(1, m) \ldots \widetilde{a}_{3, E}^{t}(l, m) \ldots\right]^{T}$,

$\left[\widetilde{a}_{2, m}^{i}\right]=\left[\begin{array}{lll}\widetilde{a}_{2, M}^{i}(1, m) \ldots & \widetilde{a}_{2, M}^{i}(l, m) \ldots\end{array}\right.$

$\left.\ldots \widetilde{a}_{2, E}^{i}(1, m) \ldots \widetilde{a}_{2, E}^{i}(l, m) \ldots\right]^{T}$

and $\left[V_{23}^{Q D}\right]$ is the diagonal matrix formed from the transmission amplitudes $\quad V_{M, 23}^{Q D}(l) \quad(43) \quad$ and $V_{E, 23}^{Q D}(l)(44)$.

Correspondingly, the binding relationships (47) and (48) in the forward direction (from QD to NP) can be represented in a matrix form as $\left[a_{3, m}^{i}\right]=\left[Z_{m}\right]\left[\widetilde{a}_{3, m}^{t}\right]$, where $\left[a_{3, m}^{i}\right]$ is the column 


$$
\begin{aligned}
& {\left[a_{3, m}^{i}\right]=\left[a_{3, M}^{i}(1, m) \ldots a_{3, M}^{i}(l, m) \ldots\right.}
\end{aligned}
$$

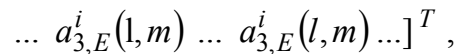

and the matrix $\left[Z_{m}\right]$ is formed from the corresponding coefficients $\quad F_{n l}^{m}, \quad \breve{G}_{n l}^{m}=m\left(k_{3} D / \sqrt{\varepsilon_{3}}\right) G_{n l}^{m} \quad$ and $\bar{G}_{n l}^{m}=-m\left(k_{3} D\right) \sqrt{\varepsilon_{3}} G_{n l}^{m}$ in (47) and (48).

The binding relationships $a_{3, M}^{r}(l, m)=V_{M, 33}^{N P}(l) a_{3, M}^{i}(l, m), a_{1, M}^{t}(l, m)=V_{M, 31}^{N P}(l) a_{3, M}^{i}(l, m)$, $a_{3, E}^{r}(l, m)=V_{E, 33}^{N P}(l) a_{3, E}^{i}(l, m), a_{1, E}^{t}(l, m)=V_{E, 31}^{N P}(l) a_{3, E}^{i}(l, m)$ and $a_{1, L}^{t}(l, m)=V_{L, 31}^{N P}(l) a_{3, E}^{i}(l, m)$ at the NP boundary can be written as $\left[a_{3, m}^{r}\right]=\left[V_{33}^{N P}\right]\left[a_{3, m}^{i}\right]$, $\left[a_{1, m}^{T}\right]=\left[V_{31, T}^{N P}\right]\left[a_{3, m}^{i}\right] \quad$ and $\quad\left[a_{1, m}^{L}\right]=\left[V_{31, L}^{N P}\right]\left[a_{3, m}^{i}\right]$, where $\left[a_{3, m}^{r}\right],\left[a_{1, m}^{T}\right]$ and $\left[a_{1, m}^{L}\right]$ are the columns

$\left[a_{3, m}^{r}\right]=\left[\begin{array}{lllll}a_{3, M}^{r}(1, m) & \ldots & a_{3, M}^{r}(l, m) & \ldots & a_{3, E}^{r}(1, m) \ldots\end{array}\right.$

$\left.\ldots a_{3, E}^{r}(l, m) \ldots\right]^{T}$,

$\left[a_{1, m}^{T}\right]=\left[a_{1, M}^{t}(1, m) \ldots a_{1, M}^{t}(l, m) \ldots a_{1, E}^{t}(1, m) \ldots\right.$

... $\left.a_{1, E}^{t}(l, m) \ldots\right]^{T}$,

$\left[a_{1, m}^{L}\right]=\left[\begin{array}{llll}a_{1, L}^{t}(1, m) & \ldots & a_{1, L}^{t}(l, m) & \ldots\end{array}\right]^{T}$,

$\left[V_{33}^{N P}\right]$ and $\left[V_{31, T}^{N P}\right]$ are the diagonal matrices formed from the reflection amplitudes $V_{M, 33}^{N P}(l)$ (52) and $V_{E, 33}^{N P}(l) \quad(54)$ and transmission amplitudes $V_{M, 31}^{N P}(l)(53)$ and $V_{E, 31}^{N P}(l)(55),\left[V_{31, L}^{N P}\right]$ is the matrix with zero "magnetic type" part and diagonal "electric type" part formed from transmission amplitudes $V_{L, 31}^{N P}(l)(56)$,

$\left[V_{31, L}^{N P}\right]=\left[\begin{array}{cccccccc}0 & \ldots & 0 & \ldots & V_{L, 31}^{N P}(1) & \ldots & 0 & \ldots \\ \ldots & \ldots & \ldots & \ldots & \ldots & \ldots & \ldots & \ldots \\ 0 & \ldots & 0 & \ldots & 0 & \ldots & V_{L, 31}^{N P}(l) & \ldots \\ \ldots & \ldots & \ldots & \ldots & \ldots & \ldots & \ldots & \ldots\end{array}\right]$

The binding relationships (69) and (70) in the backward direction (from NP to QD) can be written as $\left[\widetilde{a}_{3, m}^{i}\right]=\left[\widetilde{Z}_{m}\right]\left[a_{3, m}^{r}\right]$, where $\left[\widetilde{a}_{3, m}^{i}\right]$ is the column

$\left[\begin{array}{lll}\widetilde{a}_{3, m}^{i}\end{array}\right]=\left[\begin{array}{lll}\widetilde{a}_{3, M}^{i}(1, m) \ldots & \widetilde{a}_{3, M}^{i}(l, m) \ldots\end{array}\right.$

$\left.\ldots \widetilde{a}_{3, E}^{i}(1, m) \ldots \widetilde{a}_{3, E}^{i}(l, m) \ldots\right]^{T}$,

and the matrix $\left[\widetilde{Z}_{m}\right]$ is formed from the corresponding coefficients

$\breve{\Gamma}_{n l}^{m}=(-1)^{n+l+1} m\left(k_{3} D / \sqrt{\varepsilon_{3}}\right) G_{n l}^{m}$ $\widetilde{F}_{n l}^{m}=(-1)^{n+l} F_{n l}^{m}$, and
$\bar{\Gamma}_{n l}^{m}=(-1)^{n+l} m\left(k_{3} D\right) \sqrt{\varepsilon_{3}} G_{n l}^{m}$ in (69) and (70).

Finally, the relationships $\widetilde{a}_{3, M}^{r}(l, m)=V_{M, 33}^{Q D}(l) \tilde{a}_{3, M}^{i}(l, m)$ and $\tilde{a}_{3, E}^{r}(l, m)=V_{E, 33}^{Q D}(l) \tilde{a}_{3, E}^{i}(l, m)$ of the Mie scattering at QD can be written in a matrix form as $\left[\widetilde{a}_{3, m}^{r}\right]=\left[V_{33}^{Q D}\right]\left[\widetilde{a}_{3, m}^{i}\right]$, where

$\left[\widetilde{a}_{3, m}^{r}\right]=\left[\widetilde{a}_{3, M}^{r}(1, m) \ldots \widetilde{a}_{3, M}^{r}(l, m) \ldots\right.$

$\left.\ldots \tilde{a}_{3, E}^{r}(1, m) \ldots \tilde{a}_{3, E}^{r}(l, m) \ldots\right]^{T}$,

and $\left[V_{33}^{Q D}\right]$ is the diagonal matrix formed from the reflection amplitudes $V_{M, 33}^{Q D}(l)(72)$ and $V_{E, 33}^{Q D}(l)(73)$.

Now, it becomes possible to express multipole coefficients $\left[\widetilde{A}_{3, m}\right](80)$ of that part of the total EM field radiated by the QD+NP system, which is related to QD (i.e. sum of the field radiated initially by QD and all subsequent scattered at QD fields), in the following form:

$\left[\tilde{A}_{3, m}\right]=\left\{\mathrm{I}+\left[\xi_{m}\right]+\left[\xi_{m}\right]^{2}+\ldots+\left[\xi_{m}\right]^{n}+\ldots\right\} \times$ $\times\left[V_{23}^{Q D}\right]\left[\widetilde{a}_{2, m}^{i}\right]=\left\{\mathrm{I}-\left[\xi_{m}\right]\right\}^{-1}\left[V_{23}^{Q D}\right]\left[\widetilde{a}_{2, m}^{i}\right]$,

where I is the unit matrix and $\left[\xi_{m}\right]$ is the product of the matrices:

$\left[\xi_{m}\right]=\left[V_{33}^{Q D}\right]\left[\widetilde{Z}_{m}\right]\left[V_{33}^{N P}\right]\left[Z_{m}\right]$.

In the series (93), the term $\left[\xi_{m}\right]^{n}$ corresponds to the $n$ th-order scattering at QD of the initially radiated by the QD electromagnetic field. In the long-wave limit, when $k_{3} R_{2}, k_{2} R_{2}<<1$ and $k_{3} R_{1},\left|k_{T} R_{1}\right|,\left|k_{L} R_{1}\right|<<1$, the series (93) converges rapidly and only few first terms should be in fact taken into account.

Analogously, the multipole coefficients $\left[A_{3, m}\right]$ (81) of the total radiated EM field related to NP (i.e. the sum of all scattered at NP fields) can be expressed in the following form:

$\left[A_{3, m}\right]=\left[V_{33}^{N P}\right]\left[Z_{m}\right]\left\{\mathrm{I}-\left[\xi_{m}\right]\right\}^{-1}\left[V_{23}^{Q D}\right]\left[\widetilde{a}_{2, m}^{i}\right]$.

For the multipole coefficients $\left[A_{1, m}^{T}\right](82)$ and $\left[A_{1, m}^{L}\right]$ (83) of the fields (78) and (79) induced in the metal NP, the following expressions are valid:

$\left[A_{1, m}^{T}\right]=\left[V_{31, T}^{N P}\right]\left[Z_{m}\right]\left\{\mathrm{I}-\left[\xi_{m}\right]\right\}^{-1}\left[V_{23}^{Q D}\right]\left[\widetilde{a}_{2, m}^{i}\right]$,

$\left[A_{1, m}^{L}\right]=\left[V_{31, L}^{N P}\right]\left[Z_{m}\right]\left\{\mathrm{I}-\left[\xi_{m}\right]\right\}^{-1}\left[V_{23}^{Q D}\right]\left[\widetilde{a}_{2, m}^{i}\right]$.

In the far-field region $\widetilde{\mathbf{r}} \approx \mathbf{r}$. For this reason, the total far-field $(f-f)$ radiated by the QD+NP system (i.e. sum of the fields $\mathbf{E}_{3}^{Q D}(\widetilde{\mathbf{r}})$ (76) and $\mathbf{E}_{3}^{N P}(\mathbf{r})$ (77)) 
should be characterized by the combined multipole coefficients $\left[A_{3, m}^{f-f, Q D+N P}\right]=\left[\widetilde{A}_{3, m}\right]+\left[A_{3, m}\right]$, while the field radiated by isolated $\mathrm{QD}$ is determined by the multipole coefficients $\left[\widetilde{a}_{3, m}^{t}\right]=\left[V_{23}^{Q D}\right]\left[\widetilde{a}_{2, m}^{i}\right]$ both in near and far-field regions.

\section{Conclusions}

Thus, in this paper all the electromagnetic fields in the nonspherical "semiconductor QD + metal NP" system are consistently calculated for the case of light emission by a point (unit cell) dipole located inside QD. It enables to find the power and the rate of radiation as well as the intensity of electromagnetic energy absorption and the rate of nonradiative losses in the system under consideration. By integrating the contributions into the field radiated by QD of all QD unit cells on the base of the corresponding size-quantized exciton wave function (in line with Section 2 analysis), analogous characteristics can be found in the case of QD excitation as a whole. As a result, the luminescence efficiency change can be calculated as a function of the distance $D$ between QD and NP, radii $R_{1}$ of metal NP and $R_{2}$ of semiconductor $\mathrm{QD}$ and other parameters.

\section{Acknowledgements}

The work has been done as a part of a complex program of basic researches "Fundamental Problems of nanostructured systems, nanomaterials and nanotechnologies" NAS of Ukraine (theme \#52/7/47).

\section{Appendix}

As a result of Taylor series expansion in the integrands (35) and (36) up to the third order in $D$ and the subsequent use of normalization condition (22), the following analytical relationships between coefficients of EM field multipole expansion in two spherical coordinate systems centered at $\mathrm{O}_{1}$ and $\mathrm{O}_{2}$ points (see Fig. 1) can be obtained:

$$
\begin{aligned}
& a_{M}(l, m)=\sum_{n=0}^{3} \frac{\left(k_{3} D\right)^{n}}{n !}\left[S_{l, l \pm n}^{(m)}(D) \tilde{a}_{d, M}(l \pm n, m)+\right. \\
& \left.+\frac{m\left(k_{3} D\right)}{l(l+1)} \frac{1}{\sqrt{\varepsilon_{3}}} T_{l, l \pm n}^{(m)}(D) \widetilde{a}_{d, E}(l \pm n, m)\right], \\
& a_{E}(l, m)=\sum_{n=0}^{3} \frac{\left(k_{3} D\right)^{n}}{n !}\left[S_{l, l \pm n}^{(m)}(D) \widetilde{a}_{d, E}(l \pm n, m)-\right. \\
& \left.-\frac{m\left(k_{3} D\right)}{l(l+1)} \sqrt{\varepsilon_{3}} T_{l, l \pm n}^{(m)}(D) \widetilde{a}_{d, M}(l \pm n, m)\right],
\end{aligned}
$$

where

$$
\begin{aligned}
& S_{l, l}^{(m)}=1-\frac{\left(k_{3} D\right)^{2}}{2} \frac{2 l(l+1)-3}{4 l(l+1)-3}\left(1-\frac{m^{2}}{l(l+1)} \frac{2 l(l+1)-6}{2 l(l+1)-3}\right),(\mathrm{A} 3) \\
& S_{l, l-1}^{(m)}=\sqrt{\frac{l^{2}-1}{l^{2}} \frac{l^{2}-m^{2}}{4 l^{2}-1}} \times \\
& \times\left[1-\frac{\left(k_{3} D\right)^{2}}{2} \frac{l^{2}-3}{4 l^{2}-9}\left(1-\frac{m^{2}}{l^{2}-1} \frac{l^{2}-6}{l^{2}-3}\right)\right], \\
& S_{l, l+1}^{(m)}=-\sqrt{\frac{(l+1)^{2}-1}{(l+1)^{2}} \frac{(l+1)^{2}-m^{2}}{4(l+1)^{2}-1} \times} \\
& \times\left[1-\frac{\left(k_{3} D\right)^{2}}{2} \frac{(l+1)^{2}-3}{4(l+1)^{2}-9}\left(1-\frac{m^{2}}{(l+1)^{2}-1} \frac{(l+1)^{2}-6}{(l+1)^{2}-3}\right)\right], \\
& S_{l, l-2}^{(m)}=\sqrt{\frac{l+1}{l} \frac{(\mathrm{A} 5)}{l-1} \frac{(l-1)^{2}-m^{2}}{4(l-1)^{2}-1} \frac{l^{2}-m^{2}}{4 l^{2}-1}}, \\
& S_{l, l+2}^{(m)}=\sqrt{\frac{l}{l+1} \frac{l+3}{l+2} \frac{(l+1)^{2}-m^{2}}{4(l+1)^{2}-1} \frac{(l+2)^{2}-m^{2}}{4(l+2)^{2}-1}}
\end{aligned}
$$

$$
S_{l, l-3}^{(m)}=\sqrt{\frac{l+1}{l} \frac{l-3}{l-2} \frac{(l-2)^{2}-m^{2}}{4(l-1)^{2}-9} \frac{\left[(l-1)^{2}-m^{2}\right]\left[l^{2}-m^{2}\right]}{\left[4(l-1)^{2}-1\right]^{2}},}
$$

$$
S_{l, l+3}^{(m)}=
$$$$
=-\sqrt{\frac{l}{l+1} \frac{l+4}{l+3} \frac{(l+3)^{2}-m^{2}}{4(l+2)^{2}-9} \frac{\left[(l+2)^{2}-m^{2}\right]\left[(l+1)^{2}-m^{2}\right]}{\left[4(l+2)^{2}-1\right]^{2}}},
$$

$T_{l, l}^{(m)}=1-\frac{\left(k_{3} D\right)^{2}}{2} \frac{2 l(l+1)-1-m^{2}}{4 l(l+1)-3}$,

$$
T_{l, l-1}^{(m)}=\sqrt{\frac{l+1}{l-1} \frac{l^{2}-m^{2}}{4 l^{2}-1}}\left[1-\frac{\left(k_{3} D\right)^{2}}{2} \frac{l^{2}-2-m^{2}}{4 l^{2}-9}\right],
$$

$T_{l, l+1}^{(m)}=-\sqrt{\frac{l}{l+2} \frac{(l+1)^{2}-m^{2}}{4(l+1)^{2}-1}}\left[1-\frac{\left(k_{3} D\right)^{2}}{2} \frac{(l+1)^{2}-2-m^{2}}{4(l+1)^{2}-9}\right]$

$T_{l, l-2}^{(m)}=\sqrt{\frac{l(l+1)}{(l-2)(l-1)} \frac{(l-1)^{2}-m^{2}}{4(l-1)^{2}-1} \frac{l^{2}-m^{2}}{4 l^{2}-1}}$,

$$
T_{l, l+2}^{(m)}=\sqrt{\frac{l(l+1)}{(l+2)(l+3)} \frac{(l+2)^{2}-m^{2}}{4(l+2)^{2}-1} \frac{(l+1)^{2}-m^{2}}{4(l+1)^{2}-1}},
$$




$$
\begin{aligned}
& T_{l, l-3}^{(m)}= \\
& =\sqrt{\frac{l(l+1)}{(l-3)(l-2)} \frac{(l-2)^{2}-m^{2}}{4(l-1)^{2}-9} \frac{\left[(l-1)^{2}-m^{2}\right]\left[l^{2}-m^{2}\right]}{\left[4(l-1)^{2}-1\right]^{2}}}, \quad \text { A15) } \\
& T_{l, l+3}^{(m)}= \\
& =-\sqrt{\frac{l(l+1)}{(l+3)(l+4)} \frac{(l+3)^{2}-m^{2}}{4(l+2)^{2}-9} \frac{\left[(l+2)^{2}-m^{2}\right]\left[(l+1)^{2}-m^{2}\right]}{\left[4(l+2)^{2}-1\right]^{2}}} .
\end{aligned}
$$

Product $k_{3} D$ takes values within the range 0.02 to 0.5 for the distances $D$ between the origins of two shifted to each other spherical coordinate systems within the range $2 \ldots 10 \mathrm{~nm}, \varepsilon_{3}$ values $-1 \ldots 15$ and wavelength $\lambda_{0}-400 \ldots 800 \mathrm{~nm}$. Thus, only several terms with small $|l-n|$ values should be taken into account in fact in the sums (A1) and (A2) at such a small $D$.

\section{References}

1. N.C. Bigall, W.J. Parak, and D. Dorfs, Fluorescent, magnetic and plasmonic - Hybrid multifunctional colloidal nano objects // Nano Today 7(4), p. 282296 (2012).

2. G. Sun and J.B. Khurgin, Plasmon enhancement of luminescence by metal nanoparticles // IEEE J. Selected Topics in Quantum Electronics, 17 (1), p. 110-117 (2011).

3. A.O. Govorov, G.W. Bryant, W. Zhang, T. Skeini et al., Exciton-plasmon interaction and hybrid excitons in semiconductor-metal nanoparticle assemblies // Nano Lett. 6(5), p. 984-994 (2006).

4. J.-Y. Yan, W. Zhang, S. Duan, X.-G. Zhao and A.O. Govorov, Optical properties of coupled metalsemiconductor and metal-molecule nanocrystal complexes: Role of multipole effects // Phys. Rev. $B$, 77(16), 165301 (2008).

5. S.M. Sadeghi, Control of energy dissipation in nanoparticle optical devices: Nearly loss-free switching and modulation // J. Nanopart. Res. 14(10), p. 1184 (2012).
6. R.D. Artuso and G.W. Bryant, Hybrid quantum dot-metal nanoparticle systems: Connecting the dots // Acta Phys. Pol. 122 (2), p. 289-293 (2012).

7. H. Mertens, A.F. Koenderink, and A. Polman, Plasmon-enhanced luminescence near noblemetalnanospheres: Comparison of exact theory and an improved Gersten and Nitzan model // Phys. Rev. B, 76(11), 115123 (2007).

8. R. Knox, The Theory of Excitons. Academic Press, N.Y., 1963.

9. S.I. Pekar, The theory of electromagnetic waves in a crystal with excitons // Sov. Phys. JETP, 6, p. 785-796 (1958).

10. S.I. Pekar, Dispersion of light in the exciton absorption region of crystals // Sov. Phys. JETP, 7, p. 813-822 (1958).

11. V.V. Klimov, Nanoplasmonics: Fundamentals and Applications. PanStanford Publishing, Singapore, 2012; V.V. Klimov, Nanoplasmonics. Moscow, Fizmatlit Publ. House, 2009 (in Russian).

12. J.D. Jackson, Classical Electrodynamics. John Wiley \& Sons inc., N.Y.-London, 1962.

13. J.A. Stratton, Electromagnetic Theory. McGrawHill Book Company, N.Y.-London, 1941.

14. R. Ruppin, Decay of an excited molecule near a small metal sphere // J. Chem. Phys. 76(4), p. 16811684 (1982).

15. O. Madelung, Semiconductors other than Group IV Elements and III-V Compounds. Springer-Verlag, N.Y., 1992.

16. Al. L. Efros, M. Rosen, M. Kuno, M. Nirmal, D.J. Norris, and M. Bawendi, Band-edge exciton in quantum dots of semiconductors with a degenerate valence band: Dark and bright exciton states // Phys. Rev. B, 54(7), p. 4843-4856 (1996).

17. V.V. Datsyuk and O.M. Tovkach, Optical properties of a metal nanosphere with spatially dispersive permittivity // J. Opt. Soc. Am. B, 28 (5), p. 1224-1230 (2011); V.V. Datsyuk, A generalization of the Mie theory for a sphere with spatially dispersive permittivity // Ukr. J. Phys. 56 (2), p. 122-130 (2011). 